\title{
In vivo kinetics and nonradioactive imaging of rapidly proliferating cells in graft-versus-host disease
}

\author{
Nataliya P. Buxbaum,, Donald E. Farthing, ${ }^{1}$ Natella Maglakelidze, ${ }^{1}$ Martin Lizak, ${ }^{2}$ Hellmut Merkle, ${ }^{3}$ \\ Andrea C. Carpenter, ${ }^{4}$ Brittany U. Oliver, ${ }^{1}$ Veena Kapoor, ${ }^{1}$ Ehydel Castro, ${ }^{1}$ Gregory A. Swan, ${ }^{1}$ \\ Liliane M. dos Santos, ${ }^{5}$ Nicolas J. Bouladoux, ${ }^{5}$ Catherine V. Bare, ${ }^{1}$ Francis A. Flomerfelt, \\ Michael A. Eckhaus, ${ }^{6}$ William G. Telford, ${ }^{1}$ Yasmine Belkaid, ${ }^{5}$ Remy J. Bosselut, ${ }^{4}$ and Ronald E. Gress \\ 'Experimental Transplantation and Immunology Branch, National Cancer Institute, ${ }^{2}$ In Vivo NMR Center, National Institute \\ of Neurological Disorders and Stroke, ${ }^{3}$ Laboratory of Functional and Molecular Imaging, National Institute of Neurological \\ Disorders and Stroke, " Laboratory of Immune Cell Biology, National Cancer Institute, ${ }^{5}$ Mucosal Immunology Section, \\ National Institute of Allergy and Infectious Diseases, and ${ }^{6}$ Diagnostic and Research Services Branch, Office of the Director, \\ $\mathrm{NIH}$, Bethesda, Maryland, USA.
}

Hematopoietic stem cell transplantation (HSCT) offers a cure for cancers that are refractory to chemotherapy and radiation. Most HSCT recipients develop chronic graft-versus-host disease (cCVHD), a systemic alloimmune attack on host organs. Diagnosis is based on clinical signs and symptoms, as biopsies are risky. T cells are central to the biology of cGVHD. We found that a low Treg/CD4 ${ }^{+}$T effector memory (Tem) ratio in circulation, lymphoid, and target organs identified early and established mouse cGVHD. Using deuterated water labeling to measure multicompartment in vivo kinetics of these subsets, we show robust Tem and Treg proliferation in lymphoid and target organs, while Tregs undergo apoptosis in target organs. Since deuterium enrichment into DNA serves as a proxy for cell proliferation, we developed a whole-body clinically relevant deuterium MRI approach to nonradioactively detect cCVHD and potentially allow imaging of other diseases characterized by rapidly proliferating cells.

Conflict of interest: NPB, DEF, $\mathrm{NM}, \mathrm{BUO}, \mathrm{HM}, \mathrm{ML}$, and REG are listed on a provisional patent application (number 62414554) filed by the The National Cancer Institute and titled "Stable water isotope labeling and magnetic resonance imaging (swiMRI) for visualization of rapidly dividing cells.

Submitted: January 17, 2017

Accepted: May 16, 2017

Published: June 15, 2017

\section{Reference information:}

JCI Insight. 2017;2(12):e92851. https://doi.org/10.1172/jci. insight.92851.

\section{Introduction}

While $\mathrm{T}$ cells are at the biological forefront of diseases across many disciplines, such as autoimmunity (1-3), infectious disease $(4,5)$, inherited and acquired immunodeficiency (6), malignancy $(7,8)$, and transplantation, their in vivo behavior, encompassing generation of new cells, cell survival, and trafficking, is difficult to measure and interpret. Further complexity arises from subsets of $\mathrm{T}$ cells differing by phenotype and function. Allogeneic hematopoietic stem cell transplantation (AHSCT) is a treatment strategy widely used to cure malignant and nonmalignant diseases, for example leukemias; hemoglobinopathies, such as sickle cell disease; and inherited immunodeficiencies, such as chronic granulomatous disease. Chronic graft-versus-host disease (cGVHD) is a morbid, prevalent, and refractory AHSCT barrier (9-11), characterized by systemic immune dysregulation driven by alloreactive donor $\mathrm{T}$ cells. Tregs play a critical role in cGVHD, with a number of animal and clinical studies demonstrating their potential to prevent and treat this disease (12-15). The origin of the imbalance between regulatory and alloreactive T cells in cGVHD is not well understood, and we hypothesized that it was driven by differential in vivo kinetics of $\mathrm{CD} 4^{+} \mathrm{T}$ cell subsets.

Deuterium labeling via water or glucose for measuring cell kinetics has been used for over a decade, albeit primarily in humans. It provides an alternative to other nucleoside analogs, such as BrdU and tritiated thymidine $\left({ }^{3} \mathrm{HTdR}\right)$, that are not applicable to clinical studies due to toxicity and which incorporate into the DNA of dividing cells via the salvage pathway of nucleotide synthesis that is variable and differs by cell type (16). Indeed, T cell subsets also differ in their dependence on this pathway based on their stage of maturation, naive versus memory (17). In contrast, deuterium incorporates into cellular DNA through the constitutive de novo nucleotide synthesis pathway, which is not subject to regulation (16). Since stable isotopes are not radioactive, deuterium labeling lends itself to clinical translation with relatively small 
amounts of deuterium enrichment $(5 \%[\mathrm{v} / \mathrm{v}])$ in total body water $(\mathrm{TBW})$ required for kinetics measurements. Pioneering in vivo kinetics studies were conducted in patients with $\operatorname{HIV}(18,19)$, which measured $\mathrm{CD}^{+}$and $\mathrm{CD}^{+} \mathrm{T}$ cell kinetics, and then in many other cell types and conditions (20-30). To date, studies involving stable water isotopes measured $\mathrm{T}$ cell kinetics in circulation, a dynamic cellular compartment, while systemic cell half-lives were mathematically estimated. The logistical complication of extracting cells from organs and tissues in patients precluded consistent measurement of systemic cellular dynamics, but egress from circulation and migration into tissue is key to T cell function. These limitations are abrogated in small animal studies because cells of interest can be extracted from various organs. However, to our knowledge, no previous studies have reported using deuterium labeling to measure kinetics of $\mathrm{T}$ cell subsets in multiple compartments in small animal studies. We elucidated in vivo $\mathrm{T}$ cell subset kinetics in lymphoid and target organs in a preclinical model of cGVHD, which indeed played a role in the immune-dysregulation characteristic of cGVHD.

Once we identified which $\mathrm{T}$ cell subset predominated in the target organs (Tem) and quantified its deuterium enrichment, we then developed the deuterium MRI (dMRI) technique for detecting cGVHD in the preclinical model. To date, a major challenge in the HSCT field has been the lack of noninvasive diagnostic tools for $\mathrm{CGVHD}$, which makes initial diagnosis and continued monitoring of cGVHD severity (e.g., over the course of a clinical trial) cumbersome and relatively subjective $(31,32)$. We hypothesized and demonstrate that in vivo deuterium labeling, which results in high deuterium enrichment within rapidly proliferating $\mathrm{T}$ cells (Tem subset) following AHSCT, together with their preferential distribution to target organs, facilitate noninvasive and nonradioactive imaging of cGVHD.

\section{Results}

$C D 4^{+} T$ cell immune reconstitution in cGVHD is skewed toward distribution to target rather than lymphoid organs, with a predominance of the Tem phenotype. We employed an established minor histocompatibility antigen-mismatch cGVHD mouse model of 10.D2 $\left(\mathrm{H}^{\mathrm{d}}\right)$ BALB/c $\left(\mathrm{H} 2^{\mathrm{d}}\right)(33-36)$, where recipient mice underwent sublethal irradiation to facilitate myeolablative conditioning. This preclinical model mirrors matched unrelated donor HSCT in patients because minor antigen mismatch between donor and host induces clinical GVHD $(33,35,37)$. In the model, onset of GVHD is rapid, which differs from patients in whom manifestations are temporally separate from graft infusion and acute GVHD; however, the spectrum of clinical manifestations seen in clinical practice is well recapitulated (e.g., sclerotic dermal involvement, which is pathognomonic for cGVHD in humans; refs. 31-33, 35, 37). The major target organs affected by cGVHD in this preclinical model are skin, small and large intestines, and liver (Figure 1A). Early experiments in which congenic donor-host pairings were used (graft: Thy1.2 Ly9.2 T cell-depleted BM + Thy1.1 Ly9.2 T cells into Thy1.2 Ly9.1 hosts) showed lymphoid organs and liver T cell chimerism to be that of donor-derived peripheral (allogeneic donor spleen) phenotype.

Allogeneic graft recipients consistently developed cGVHD by posttransplant day +28 , while at day +14 , the animals appeared clinically normal (36) (Supplemental Figure 1; supplemental material available online with this article; https://doi.org/10.1172/jci.insight.92851DS1). However, lymphoid atrophy and low absolute $\mathrm{CD}^{+} \mathrm{T}$ cell numbers in the thymi and lymph nodes were observed in allogeneic recipients at day +14 and persisted through day +28 . In addition, thymi of allogeneic recipients contained a mature $\left(\mathrm{CD} 44^{\text {high }}\right) \mathrm{CD}^{+} \mathrm{T}$ cell infiltrate with a marked reduction in $\mathrm{T}$ cell precursors (Supplemental Figure 2). At day +14 , splenomegaly was seen in allogeneic recipients. It was characterized by mild extramedullary hematopoiesis and a high number of $\mathrm{CD} 4^{+} \mathrm{T}$ cells, primarily of the Tem $\left(\mathrm{CD} 4^{+} \mathrm{FoxP} 3^{-} \mathrm{CD} 44^{\text {high }} \mathrm{CCR}^{-}\right)$ phenotype (Supplemental Figure 3, A and B). Following initial size increase, the spleen underwent atrophy with a near absence of $\mathrm{CD}^{+} \mathrm{T}$ cells in the spleen at day +28 (Supplemental Figure 3A).

The target organs affected by cGVHD (e.g., integument, small intestine, and liver) were characterized by lymphocytic infiltrates (Supplemental Figure 3C and Supplemental Figure 4, A-J). Liver parenchyma of allogeneic recipients contained approximately a log higher absolute number of $\mathrm{CD}^{+} \mathrm{T}$ cells by day +14 compared with the syngeneic counterparts (Supplemental Figure 3C). Similar to other target organs affected by cGVHD, the predominant CD4 ${ }^{+} \mathrm{T}$ cell subset was Tem, and a decreased Treg $\left(\mathrm{CD} 4{ }^{+} \mathrm{FoxP} 3^{+} \mathrm{CD} 25^{+}\right) /$ Tem ratio was observed (Supplemental Figure 3D). Dermal parenchyma sections of the allogeneic cohort had higher $\mathrm{CD}^{+}$absolute counts than syngeneic counterparts (Supplemental Figure 5, A and B). Furthermore, Tem phenotype predominated in the dermal $\mathrm{CD} 4^{+} \mathrm{T}$ cell pool in the allogeneic setting both at day +14 and day +28 (Supplemental Figure 5, C and D). In the small intestine, $\mathrm{CD}^{+} \mathrm{T}$ cell number was 

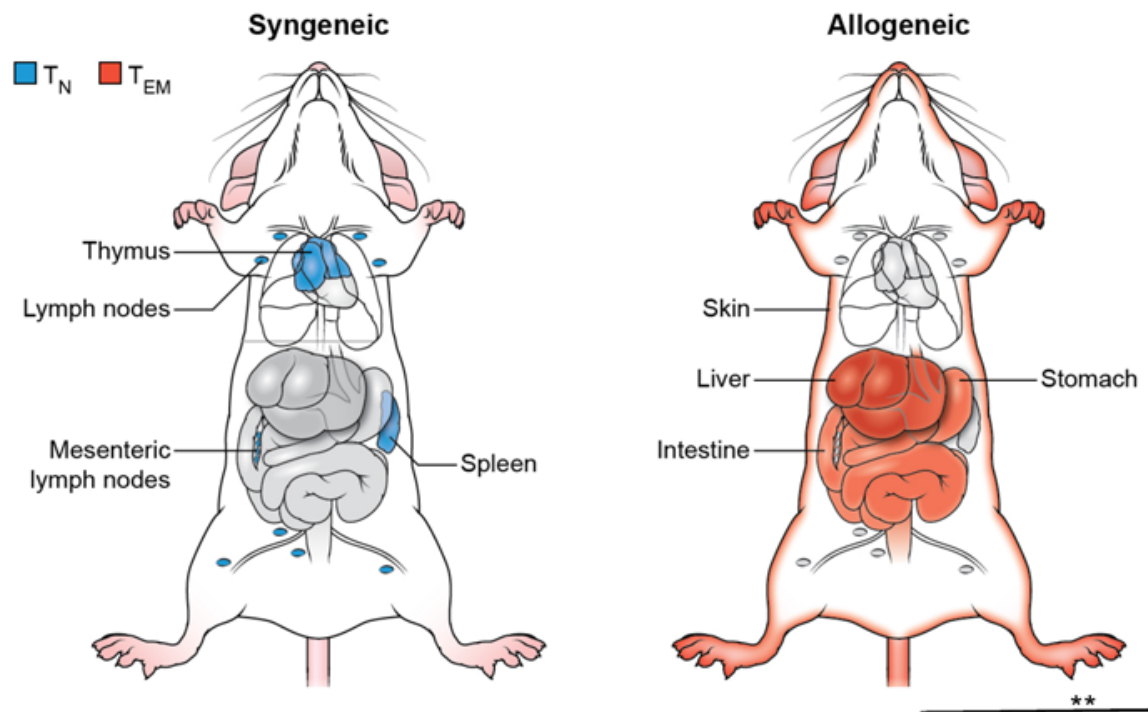

B

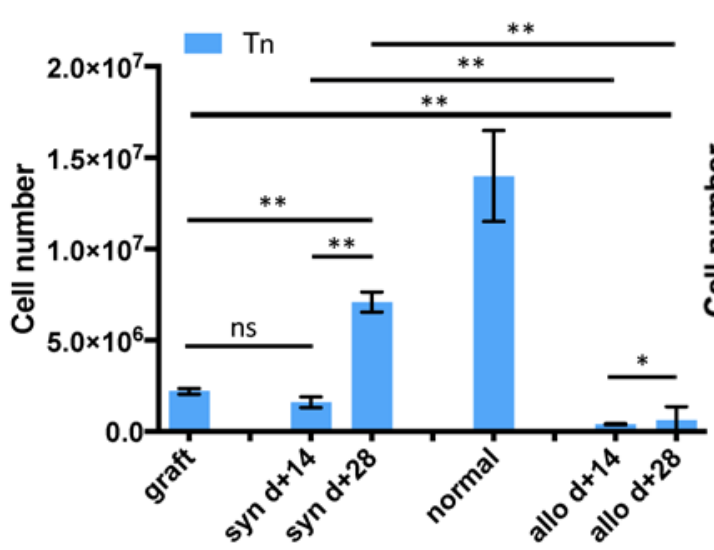

D

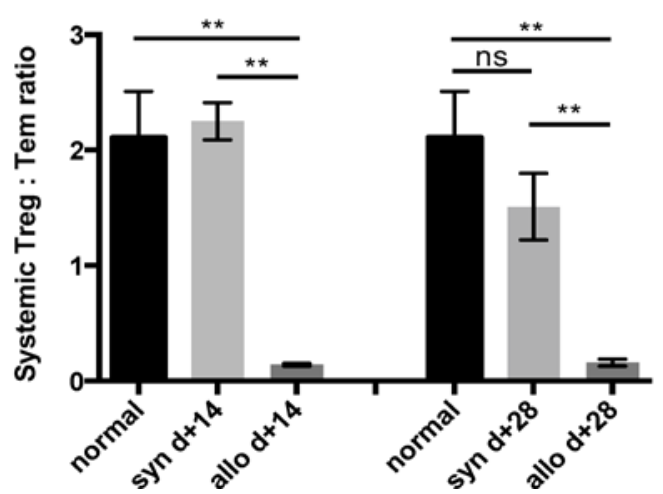

C

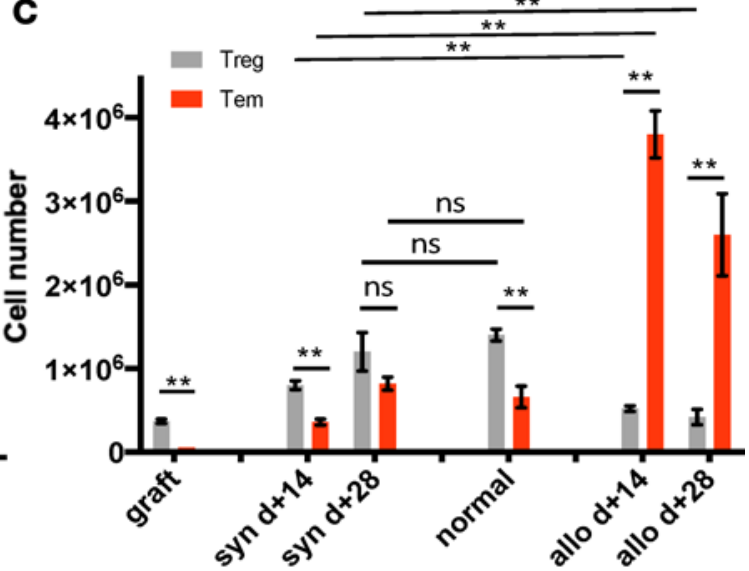

E

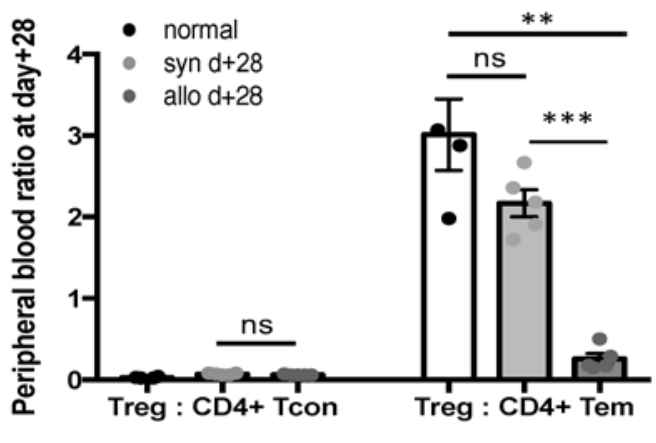

Figure 1. CD4 ${ }^{+} \mathrm{T}$ cell pool size, composition, and distribution for normal mice and syngeneic (syn) and allogeneic (allo) hematopoietic stem cell transplant recipients. CD4 ${ }^{+} \mathrm{T}$ cell numbers extracted from spleen, parenchymal liver, small intestine, flank skin, and blood were combined. (A) Graphic representation of the predominant $\mathrm{CD} 4^{+} \mathrm{T}$ cell subset distribution in syngeneic and allogeneic recipients at day +28 . In the syngeneic setting, $\mathrm{CD} 4^{+}$ T cells primarily found in lymphoid organs and of Tn phenotype. In the allogeneic setting, CD4+ $T$ cells were primarily in target tissues (skin, liver, gut) and of Tem phenotype. (B) The mean total Tn cell number within the graft, normal mice, and syngeneic and allogeneic recipients at day +14 and +28 . (C) The mean total Tem and Treg numbers within the graft, normal mice, and syngeneic and allogeneic recipients at day +14 and +28 . (D) Treg/Tem ratio was $>1$ in the graft, syngeneic recipients, and normal mice, while it was $<1$ in cGVHD setting. (E) Treg/CD4 ${ }^{+}$TCON ratio vs. Treg/CD4+ Tem ratio for normal mice, and syngeneic and allogeneic recipients at day +28 in blood. Data are representative of more than 3 independent experiments. For panels B-E, $n=10 ;{ }^{* *} P<0.01,{ }^{* *} P<0.001$; Tukey's test with 2-way ANOVA. 
increased in the lamina propria (LP) and intraepithelial (IE) compartment of allogeneic compared with syngeneic recipients (Supplemental Figure 6, A, B, D, E). Furthermore, the Tem proportion and total number were significantly higher in allogeneic LP than syngeneic and normal LP by day +28 , and in the IE compartment at both time points (Supplemental Figure 6, C, F, G).

Overall, $\mathrm{CD}^{+} \mathrm{T}$ cell immune reconstitution in the syngeneic transplant setting mirrored distribution and composition of $\mathrm{T}$ cells in normal mice, e.g., lymphoid organs (Figure 1A). The predominant phenotype for $\mathrm{CD}^{+} \mathrm{T}$ cells in normal mice and syngeneic recipients was naive $\left(\mathrm{T}_{\mathrm{N}}, \mathrm{FoxP}^{-} \mathrm{CD} 44^{\text {low }} \mathrm{CCR} 7^{+}\right)$ (Figure 1, A and B). In the syngeneic cohort, few $\mathrm{CD}^{+} \mathrm{T}$ cells were found outside of the lymphoid tissues. In contrast, lymphoid organs in the allogeneic setting underwent atrophy, with few $\mathrm{CD} 4^{+} \mathrm{T}$ cells present in these anatomic locations. Additionally, in the allogeneic cohort, $\mathrm{CD} 4^{+} \mathrm{T}$ cells were primarily localized to the target tissues, such as the integument, gastrointestinal tract, and liver and were mostly of the Tem phenotype (Figure 1, A and C). This resulted in a very low $(<<1)$ target organ, systemic, and peripheral blood Treg/Tem ratio in the allogeneic setting (Figure 1, D and E). In contrast, the Treg/Tem ratio was significantly higher $(>1)$ in the syngeneic and normal cohorts in all the same sites (Figure $1, \mathrm{D}$ and $\mathrm{E})$. To elucidate why Tregs were diminished while Tem cells were expanded in the allogeneic setting, we obtained measurements of in vivo cell kinetics for $\mathrm{CD} 4^{+} \mathrm{T}$ cell subsets.

In vivo Treg kinetics indicate marked proliferation in lymphoid and target organs and diminished survival in target organs. To quantify Treg expansion in lymphoid and target organs, spleen, and liver, we applied in vivo deuterated $\left({ }^{2} \mathrm{H}_{2} \mathrm{O}\right)$ water labeling to $5 \%(\mathrm{v} / \mathrm{v})$ TBW (Figure 2, A and B). Then, we extracted T cell subsets from each organ at the conclusion of each sequential labeling period. Cellular DNA was purified and enzymatically digested to nucleosides. Subsequently, we compared the deuterium enriched fraction of deoxyadenosine $(\mathrm{dA}[\mathrm{M}+1])$ to unenriched $\mathrm{dA}$, which allowed calculation of the fraction of new Tregs generated during each labeling period (38). Deuterium enrichment in all 4 DNA nucleosides: $\mathrm{dA}$, deoxythymidine $(\mathrm{dT})$, deoxycytosine $(\mathrm{dC})$, or deoxyguanine $(\mathrm{dG})$, can be measured; however, we selected a single nucleoside to allow high throughput analysis, and dA was chosen because DNA of rodents and humans has a higher dA-dT content than dG-dC $(39,40)$, allowing superior sensitivity of detection when evaluating a small number of cells, as low as 10,000 per analysis (38). As previously shown, ${ }^{2} \mathrm{H}_{2} \mathrm{O}$ in TBW incorporates into DNA nucleosides through the de novo nucleotide synthesis pathway (16). When TBW enrichment of $5 \%(\mathrm{v} / \mathrm{v})$ is maintained for at least a week, the dA of highly proliferative cells will enrich to $\sim 20 \% \mathrm{dA}$ $(\mathrm{M}+1)$ (Figure 2, $\mathrm{C}$ and $\mathrm{D})$. In our studies, the labeling periods were selected to cover biologically relevant time points in the model (Figure 2B and Supplemental Figure 1A), day +7 to $+14,+14$ to $+21,+21$ to +28 , and +28 to +35 , with measurements obtained on day $+14,+21,+28$, and +35 (Figure $2 \mathrm{~B}$ ). Similarly, delabeling studies were conducted by labeling to $5 \%(\mathrm{v} / \mathrm{v})$ TBW during day +7 to +14 , obtaining dA $(\mathrm{M}+1)$ measurement at day +14 , and then discontinuing the labeling and measuring $\mathrm{dA}(\mathrm{M}+1)$ on days $+21,+28$, and +35 (Figure $2 \mathrm{~B}$ ).

Tregs extracted from the spleen and liver parenchyma showed high rates of label gain for allogeneic and syngeneic cohorts (Figure 3, A and B). Furthermore, in the allogeneic setting, Tregs experienced label gain rates similar to those of Tem cells (Figure 3, C and D). While both subsets had similarly high rates of label gain in lymphoid and target organs, this did not result in an increased systemic Treg cell number in allogeneic mice, while the Tem cells were abundant (Figure 1C). This difference in absolute numbers despite similar label gain kinetics could be driven by differential cell loss kinetics. Indeed, our kinetics data show that Treg cell loss from the liver exceeds that of the Tem subset (Figure 4A). Moreover, a key difference between the allogeneic liver Treg and Tem subsets was the presence of Caspase 3 staining (Figure 4B), which indicated greater propensity for apoptosis. When first-order elimination kinetics were applied to the observed label loss rates, Treg subset half-life in liver was estimated to be roughly half of the Tem half-life. Increased loss of Tregs in the target tissues may explain why Tregs as a subset did not expand following HSCT in the allogeneic recipients, despite their high rates of label gain. Tregs were not observed in target organs nor circulation in an appreciable number throughout the course of allogeneic immune reconstitution, while Tem cells were circulating and predominated in the target organs (Figure 1, A and C, and Figure 4, C and D).

In vivo Tem kinetics indicate marked expansion in lymphoid and target organs and suggest trafficking to target organs. In allogeneic recipients, the total number of Tem cells in all evaluated tissues combined, e.g., spleen, liver, small intestine, skin, peripheral blood, and lymph nodes, approached 4 million by day +14 , while only 0.06 million were contained in the graft (Figure 1C). This represents more than a 60 -fold expansion of this compartment. In comparison, in syngeneic recipients, the Tem compartment expanded 


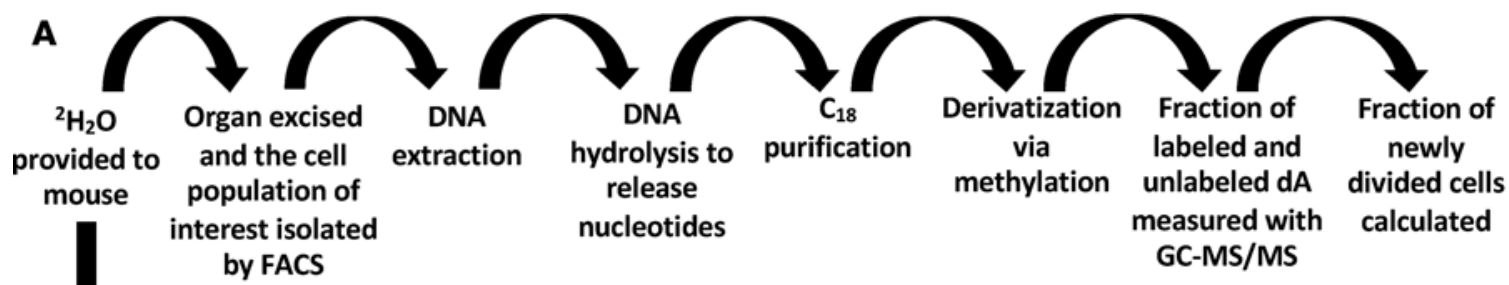

Deuterium
MRI (dMRI)

B

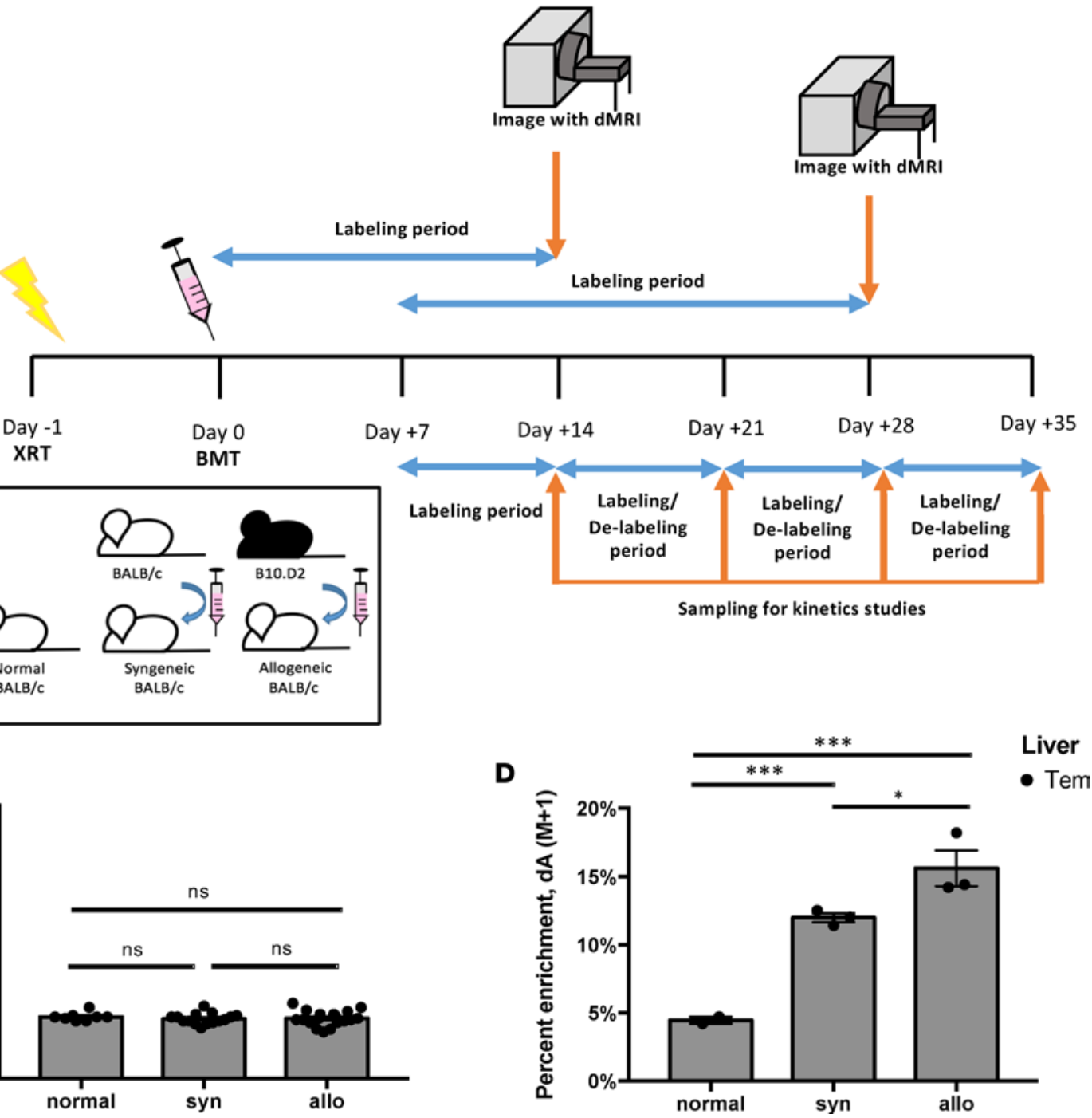

Figure 2. Experimental schema for hematopoietic stem cell transplantation (HSCT), deuterated water labeling, in vivo cell kinetics measurements, and deuterium MRI (dMRI) labeling imaging approach. (A) Overview of method for deuterium water labeling of DNA. (B) Pictorial representation of syngeneic (syn) and allogeneic (allo) HSCT, timeline for ${ }^{2} \mathrm{H}_{2} \mathrm{O}$ labeling and delabeling periods for cell kinetics studies, and ${ }^{2} \mathrm{H}_{2} \mathrm{O}$ labeling for subsequent dMRI imaging of transplant recipients. (C) ${ }^{2} \mathrm{H}_{2} \mathrm{O}$ levels achieved in total body water (TBW) in normal mice and syngeneic and allogeneic $\mathrm{HSCT}$ recipients following 14 and 21 days of ${ }^{2} \mathrm{H}_{2} \mathrm{O}$ labeling prior to dMRI imaging at day +14 and +28 after HSCT, respectively. Data for 9 independent experiments were pooled ( $n=8$ mice for normal, $n=15$ mice for syngeneic cohort, $n=19$ mice for allogeneic cohort). (D) Deuterium enrichment in deoxyadenosine (dA) measured as dA $(M+1)$ via GC-MS/MS for liver CD4+ Tem subset in the 3 cohorts at day $+14\left(n=3\right.$ for each cohort). For panels $\mathbf{C}$ and $\mathbf{D}$, ${ }^{*} P<0.05$, ${ }^{* * *} P<0.001$; Tukey's test with 2-way ANOVA.

roughly 10-fold (Figure 1C). In allogeneic recipients, label gain for the Tem subset in the spleen and liver was robust through day +14 (Figure $5, \mathrm{~A}$ and $\mathrm{B}$ ) and paralleled the overall concurrent increase in the Tem cell number during this period in both organs (Supplemental Figure 3, B and D). Additionally, the Tem subset in both organs did not show significant Caspase 3 staining (Figure 4B). Label loss in the Tem liver 
A

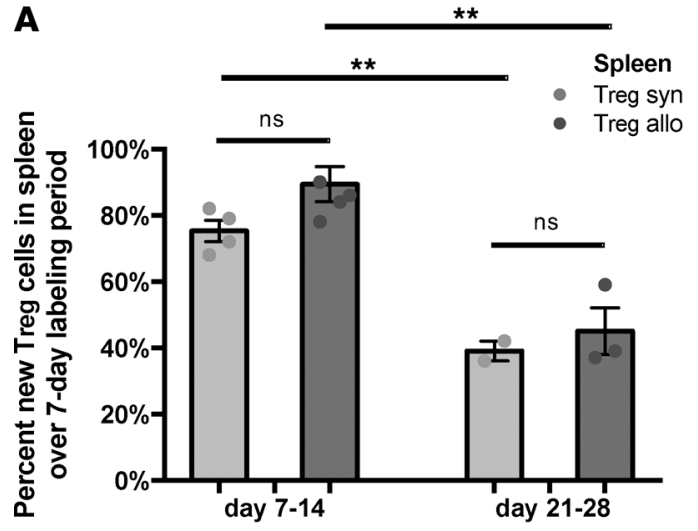

Post HSCT period

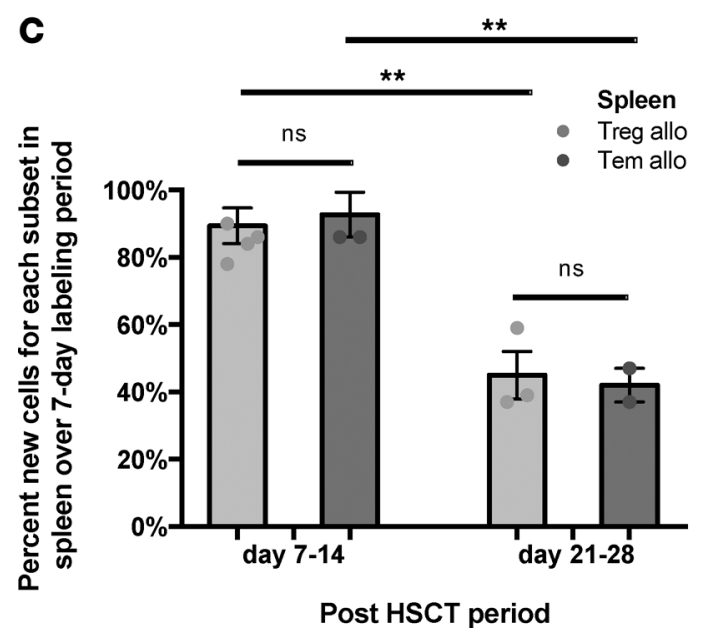

B

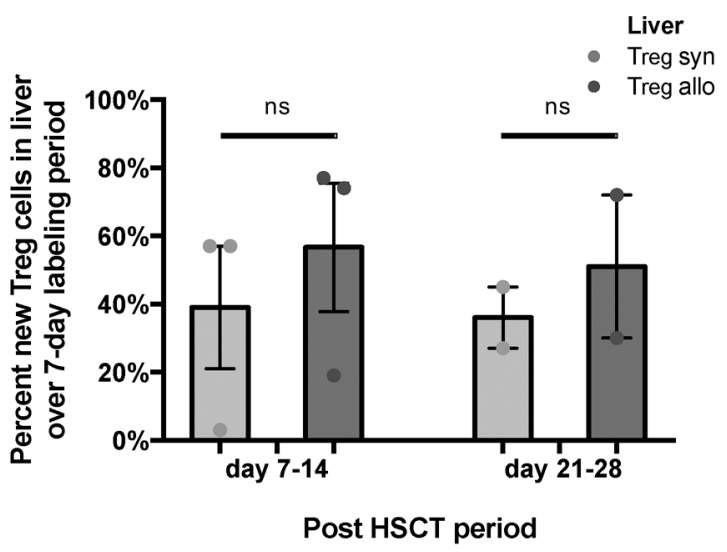

D

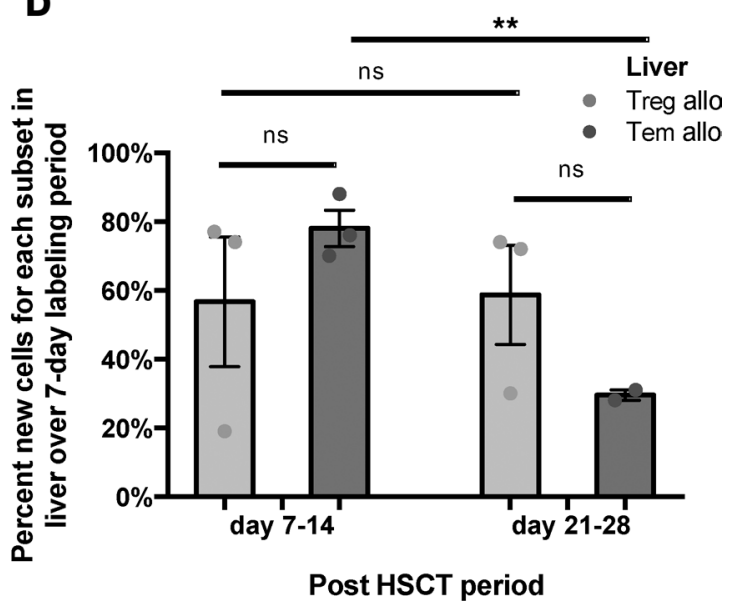

Figure 3. Cell gain kinetics for Tregs in the spleen and liver for allogeneic (allo) and syngeneic (syn) hematopoietic stem cell transplant recipients (HSCT). (A) Percentage of new Tregs in the spleen for each cohort at day +14 and +28 , following 7 days of labeling. Data for 5 independent experiments pooled for day +7 to +14 analysis, and 3 independent experiments for day +21 to +28 analysis. (B) Percentage of new Tregs in the liver for each cohort measured at day +14 and +28 , following 7 days of labeling. Data for 3 independent experiments were pooled for day +7 to +14 analysis and 2 independent experiments for day +21 to +28 analysis. (C) Percentage of new Treg and Tem cells in the allogeneic spleen measured at day +14 and +28 , following 7 days of labeling. Data for 5 independent experiments pooled for the day +7 to +14 analysis, and 3 independent experiments for day +21 to +28 analysis. (D) Percentage of new Treg and Tem cells in the allogeneic liver measured at day +14 and +28 , following 7 days of labeling. Data for 3 independent experiments pooled for day +7 to +14 analysis, and 2 independent experiments for day +21 to +28 analysis. For panels $\mathbf{A}$ and $\mathbf{C}, n=2-7$ mice; for panels $\mathbf{B}$ and $\mathbf{D}, n=5-8$ mice; ${ }^{* *} P<0.01$; Tukey's test with 2-way ANOVA.

subset was slower than that of Tregs (Figure 4A), while spleen Tem cells appeared to undergo rapid label loss (Figure 5C). The mechanism for Tem subset label and absolute number loss from the spleen may be trafficking. This was supported by the measurement of a high number of Tem cells in circulation of allogeneic recipients at day +14 (Figure 4D). Tem in vivo cell kinetics showed: i) high label gain in lymphoid and target organs; ii) rapid loss from the lymphoid organ concurrent with a high circulating number; iii) target organ infiltration; and iv) no increased propensity for apoptosis. Robust expansion of the Tem subset in the allogeneic setting, both in absolute number and with regard to prominent label gain, contributed to the extremely low $(<<1)$ Treg/CD4 $4^{+}$Tem ratio in circulation, lymphoid, and target organs, early in cGVHD pathogenesis $($ day +14$)$ and in fully expressed cGVHD $($ day +28$)$.

Deuterated water labeling followed by in vivo dMRI distinguishes mice with cGVHD from controls. Our measurements of high deuterium $\left({ }^{2} \mathrm{H}\right)$ enrichment in dA of liver-infiltrating Tem cells (Figure 2D) and the high number of this cell type within target organs of cGVHD-affected mice (Figure 1A) prompted us to hypothesize that measuring in vivo ${ }^{2} \mathrm{H}$ signal in target organs could allow us to distinguish cGVHD-affected mice from syngeneic controls. Given the difference in ${ }^{2} \mathrm{H}$ levels between TBW enrichment of $5 \%$ (v/v) achieved for kinetics studies and the resultant higher enrichment $(\sim 15 \%-20 \%)$ in the DNA of liv- 


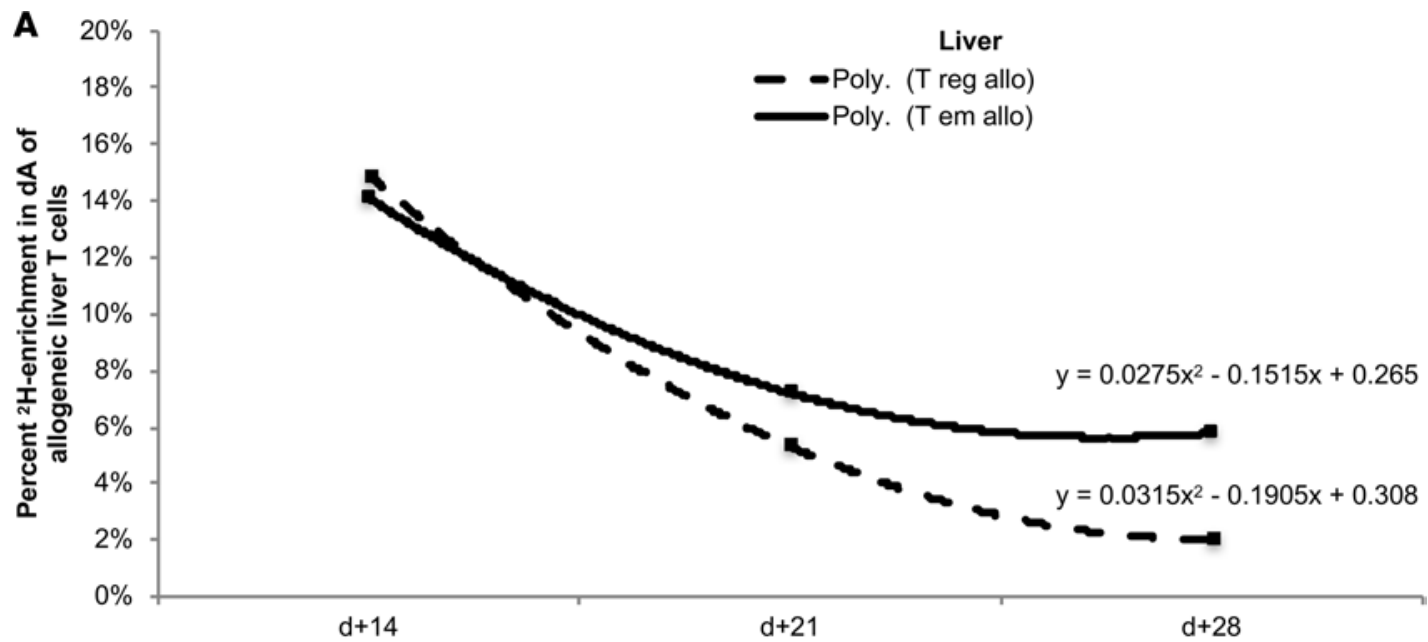

B

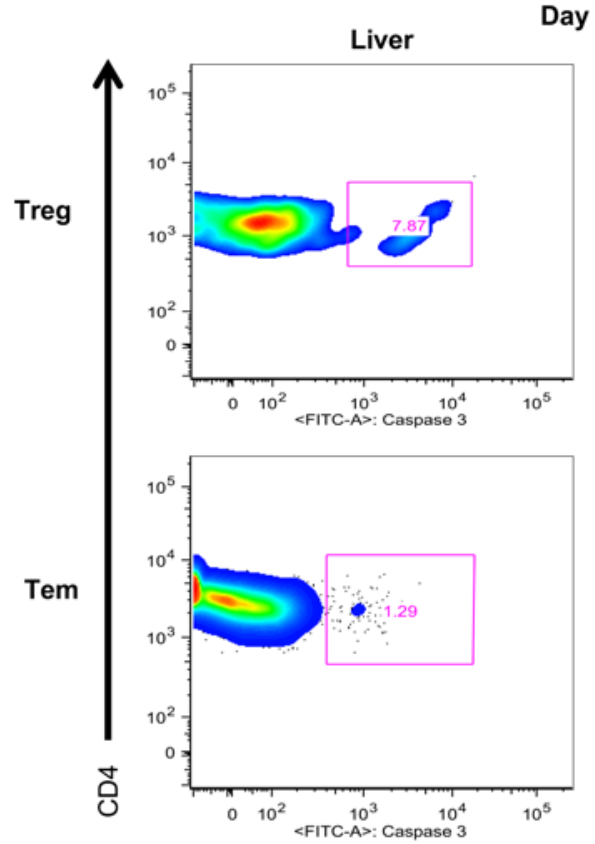

Day post HSCT

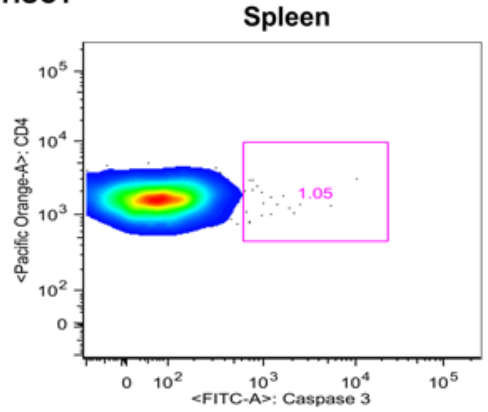

Caspase 3

C

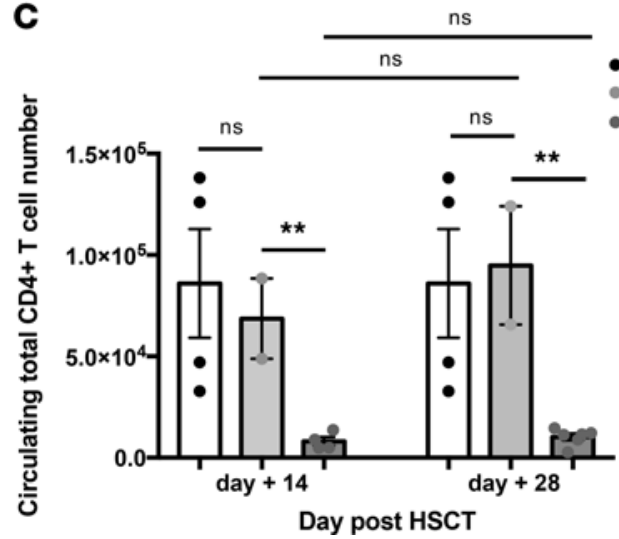

D

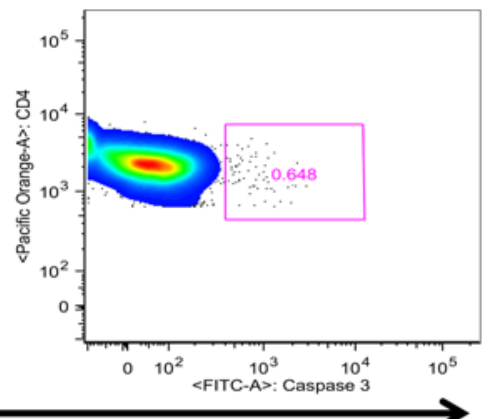

D

\section{normal}

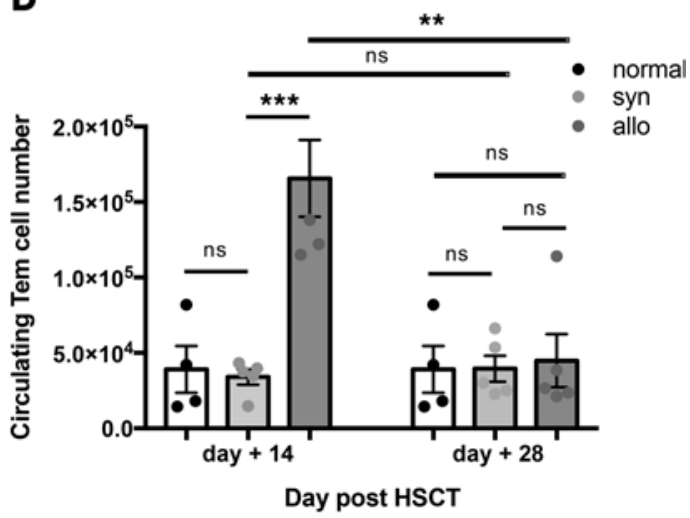

Figure 4. Label loss kinetics for Tregs in the liver in allogeneic (allo) hematopoietic stem cell transplant recipients (HSCT) are driven by increased propensity for apoptosis rather than trafficking. (A) Label loss kinetics for allogeneic liver-derived Treg and Tem cells. Data are representative of 3 independent experiments for day +14 and +28 ( $n=5-8$ mice per experiment per cohort). (B) Caspase 3 staining for CD4 ${ }^{+}$Treg and Tem subsets extracted from the spleen and liver at day +14 ( $n=5$ mice per cohort). (C) The mean total CD4+ T cell number measured in the peripheral blood of each experimental cohort at day +14 and +28 . (D) The mean total Tem cell number measured in the peripheral blood of each experimental cohort at day +14 and +28 . Data are representative of 4 independent experiments ( $n=2-6$ mice per experiment per cohort). For panels $\mathbf{C}$ and $\mathbf{D}$, ${ }^{* *} P<0.01,{ }^{* * *} P<0.001$; Tukey's test with 2-way ANOVA. 
A

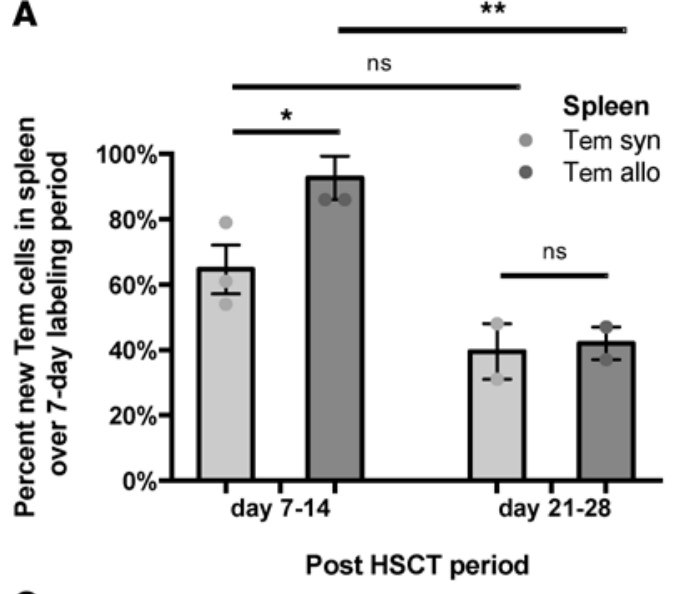

B

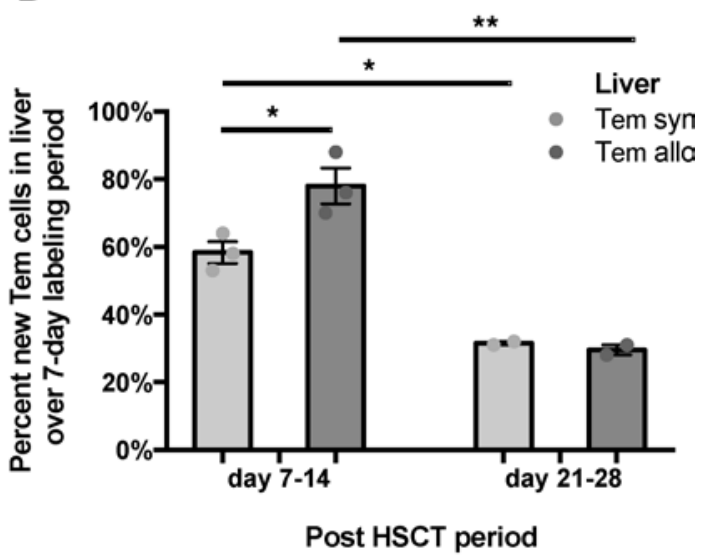

C

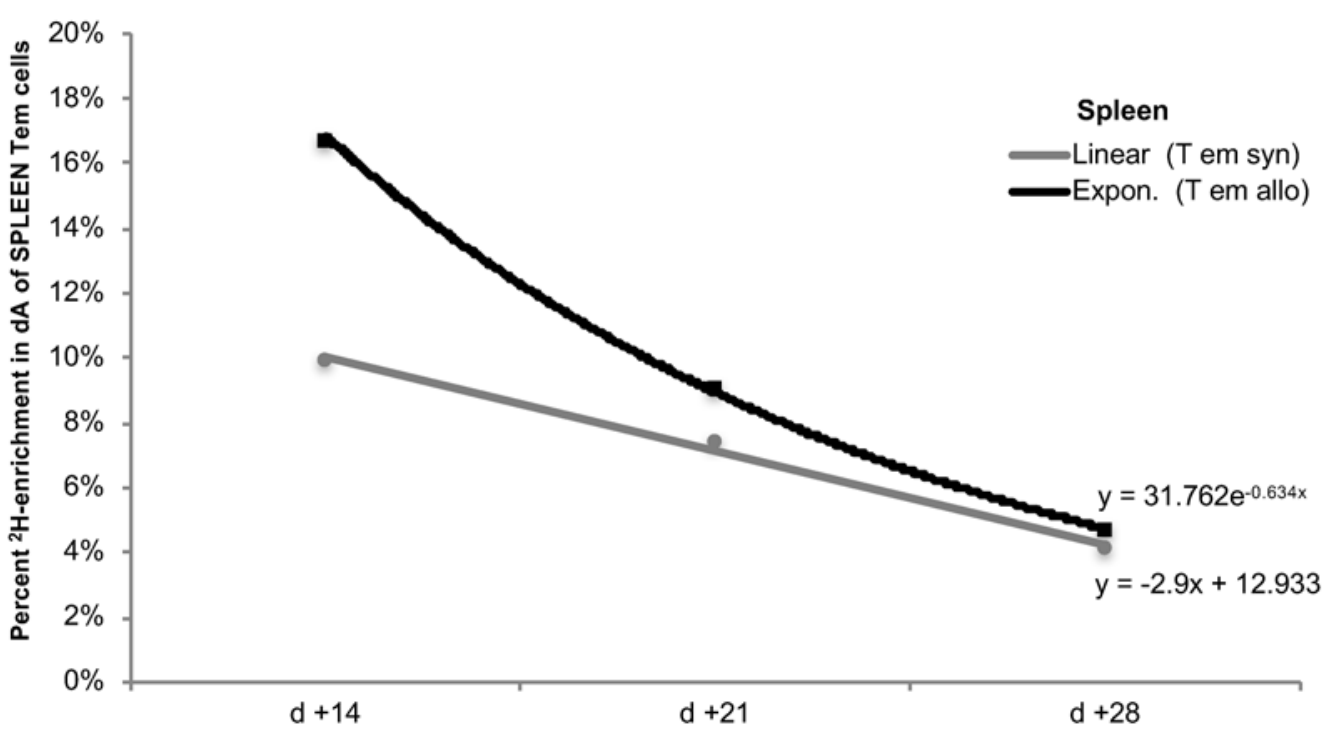

Day post HSCT

Figure 5. CD4+ Tem in vivo cell kinetics show robust cell gain in the spleen and liver in allogeneic (allo) hematopoietic stem cell transplant recipients (HSCT), with rapid concurrent label loss kinetics in the spleen and increased number in circulation. (A) Percentage of new Tem cells in the spleen for each experimental cohort measured at day +14 and +28 , following 7 days of labeling for each time point. Data for 5 independent experiments were pooled for the day +7 to +14 analysis (each independent experiment represents a pooled sample from $n=2-7$ mice), and 3 independent experiments for day +21 to +28 analysis. (B) Percentage of new Tem cells in the liver for each experimental cohort measured at day +14 and +28 , following 7 days of labeling for each time point. Data for 3 independent experiments were pooled for the day +7 to +14 analysis (each independent experiment represents a pooled sample from $n=5-8$ mice), and 2 independent experiments for day +21 to +28 analysis. (C) Label loss kinetics for Tem cells from spleens of allogeneic versus syngeneic recipients. Data are representative of 2 independent experiments (each independent experiment represents a pooled sample from $n=2-7$ mice). Data are representative of 4 independent experiments ( $n=2-6$ mice per experiment per cohort). For panels $\mathbf{A}$ and $\mathbf{B},{ }^{*} P<0.05,{ }^{* *} P<0.01$; Tukey's test with 2-way ANOVA.

er-infiltrating Tem subset, we approached nuclear magnetic resonance/MRI (NMR/MRI) physicists to manufacture a probe that could detect ${ }^{2} \mathrm{H}$ by MRI in small animals. We first used phantoms (vials) containing various concentrations of ${ }^{2} \mathrm{H}_{2} \mathrm{O}(0.015 \%-50 \%)$ in $\mathrm{H}_{2} \mathrm{O}(\mathrm{v} / \mathrm{v})$ to evaluate sensitivity of ${ }^{2} \mathrm{H}$ detection by MRI, using an ${ }^{2} \mathrm{H}$ probe that could accommodate small samples (vials). We then used the same probe to detect ${ }^{2} \mathrm{H}$ in DNA extracted from mouse tumor cells that were cultured in vitro in ${ }^{2} \mathrm{H}_{2} \mathrm{O}$-containing media to demonstrate feasibility of ${ }^{2} \mathrm{H}$ MRI detection where ${ }^{2} \mathrm{H}$ was bound to DNA (data not shown). Then, the ${ }^{1} \mathrm{H}-{ }^{2} \mathrm{H}$ coil for a 9.4 Tesla magnet was manufactured at the NIH for small animal imaging, which commenced in the cGVHD preclinical model (Supplemental Figure 7, A-D). Thus, we developed, to our knowledge, a novel MRI-based imaging modality, dMRI. As predicted, the detected ${ }^{2} \mathrm{H}$ signal within livers of cGVHD-affected mice was significantly greater than that measured in syngeneic controls at day +28 , following 21 days of ${ }^{2} \mathrm{H}_{2} \mathrm{O}$ labeling to TBW of $5 \%$ (v/v) (Figure 6, A-D, and G). For mouse imaging, each subject was imaged with an attached phantom containing $5 \%{ }^{2} \mathrm{H}_{2} \mathrm{O}$ in $\mathrm{H}_{2} \mathrm{O}(\mathrm{v} / \mathrm{v})$, which served an internal 
reference and normalization parameter. The ${ }^{2} \mathrm{H}$ signal from each organ was compared with the reference and was reported as normalized deuterium signal (nDS). ${ }^{1} \mathrm{H}$ (proton) signal was used for anatomical imaging (traditional MRI), and the ${ }^{2} \mathrm{H}$ signal was overlaid on the reference image, allowing us to report on the ${ }^{2} \mathrm{H}$ signal corresponding to the organ, e.g., liver, small bowel, etc. (Figure 6, A-D). Subsequent experiments showed that significant differences between allogeneic and syngeneic liver nDS were also detected at day +14 , following 14 days of labeling to $5 \%(\mathrm{v} / \mathrm{v}){ }^{2} \mathrm{H}_{2} \mathrm{O}$ in TBW, albeit at lower nDS values than those measured at day +28 , which followed a longer labeling period ( 21 days; Figure $6 \mathrm{E}$ ). At day +14 , allogeneic mice had no clinical symptoms of cGVHD and were well-appearing (Supplemental Figure 1A). Thus, dMRI allowed noninvasive in vivo imaging of cGVHD in target organs ahead of animals becoming ill-appearing. Furthermore, no difference in ${ }^{2} \mathrm{H}$ signal was observed within a tissue not targeted by cGVHD in our mouse model, such as the quadriceps muscle group, between syngeneic and allogeneic cohorts at both time points (Figure 6, F and $\mathrm{H}$ ).

\section{Discussion}

cGVHD is a major barrier to successful HSCT, with high prevalence, morbidity, and mortality $(10,11)$. To date, clinical diagnosis and monitoring of cGVHD progression or response to therapy have been challenging $(31,32)$, with no clinically validated biomarker; the ability to obtain biopsies of affected organs has been limited by accessibility and morbidity concerns, particularly for pediatric patients and for certain sites, such as the liver, regardless of age. To address this clinical need, we developed a noninvasive and nonradioactive imaging modality, dMRI. First, we determined that the Treg/CD4 ${ }^{+}$Tem ratio $<<1$, in target organs, lymphoid organs, and blood as a key parameter altered early in cGVHD pathogenesis, which is then maintained through fully established cGVHD in a preclinical model. Measurement of the Treg/CD4 $4^{+}$Tem ratio prospectively on HSCT recipients may identify patients at high risk for cGVHD. Additional rationale for this parameter to be measured prospectively is that it may be a biomarker of ongoing disease (41) and/or could potentially be used as a marker of response to therapy. The ratio we identify here underscores that subsets of non-Tregs, which are often treated as a single cell population and referred to as $\mathrm{T}$ conventional (TCON) cells $(12,13,42-44)$, have differential in vivo function, behavior, and phenotype. For instance, in syngeneic recipients, $\mathrm{CD} 4{ }^{+} \mathrm{TCON}$ cells are primarily of naive phenotype, while in the allogeneic recipients $\mathrm{CD}^{+}{ }^{+} \mathrm{TCON}$ are predominantly Tem. Indeed, the Treg/CD4 ${ }^{+}$Tem ratio is superior to the Treg/TCON ratio $(12,13)$ and the Treg/CD4 ${ }^{+}$TCON ratio $(42,45)$, which are widely reported on HSCT and other fields, as we did not find these to be consistently altered across relevant disease sites - lymphoid, target, and circulation - nor are they currently being used in clinical staging of GVHD in patients (31).

To understand the biological basis for the imbalance between immune regulation and activation in cGVHD, we measured in vivo dynamic behavior of Treg and CD4 ${ }^{+}$Tem cells in target and lymphoid organs of cGVHD over the course of disease progression. Quantitative dynamic in vivo cell kinetics measurements have not been previously obtained for Tregs or subsets of non-Treg CD4 ${ }^{+} \mathrm{T}$ cells (12, 46-49). Additionally, we measured these processes in multiple anatomic compartments. Previous studies employed mathematical modeling to hypothesize in vivo cell kinetics outside of the circulation while measuring deuterium label gain and loss in circulating cells $(18,19,21,22,24,27,30,50-54)$. In contrast, we obtained quantitative measurements of deuterium gain and loss in T cell subsets from lymphoid and target organs, and we found the same subset (i.e., Treg) to behave differently in the spleen compared with the liver. Bioluminescent studies in mice have demonstrated differential trafficking of T cell subsets in acute GVHD (43, 44, 48, 49); however, this methodology is based on enumeration of cells in organs, which we accomplished by an alternate method of direct extraction from tissues and subset phenotyping by flow cytometry. Furthermore, enumeration does not address the dynamic behavior of cells in vivo, since minimal $\mathrm{T}$ cell subset accumulation in a particular compartment (e.g., Treg in liver) could be due to diminished proliferation or robust proliferation followed by apoptosis. Furthermore, in vivo kinetics of Tregs have not been previously compared with those of Tem (47-49). Other studies evaluated lymphoid organs (46), but lymphoid manifestations of GVHD do not provide a full view of in vivo biology (55). For instance, in our model, we show that lymphoid and target organs are different with respect to $\mathrm{T}$ cell number, subset proportions, and cell kinetics.

We found that impaired Treg survival in target organs, rather than compromised proliferative capacity of this subset as described by others $(56,57)$, underlies the reduced Treg/Tem ratio. We show that CD4 ${ }^{+}$ Tem and Treg cells undergo robust expansion in lymphoid and target tissues. Therapy for GVHD has aimed to reduce general $\mathrm{T}$ cell proliferation, while our findings indicate that both immunoregulatory and 
A

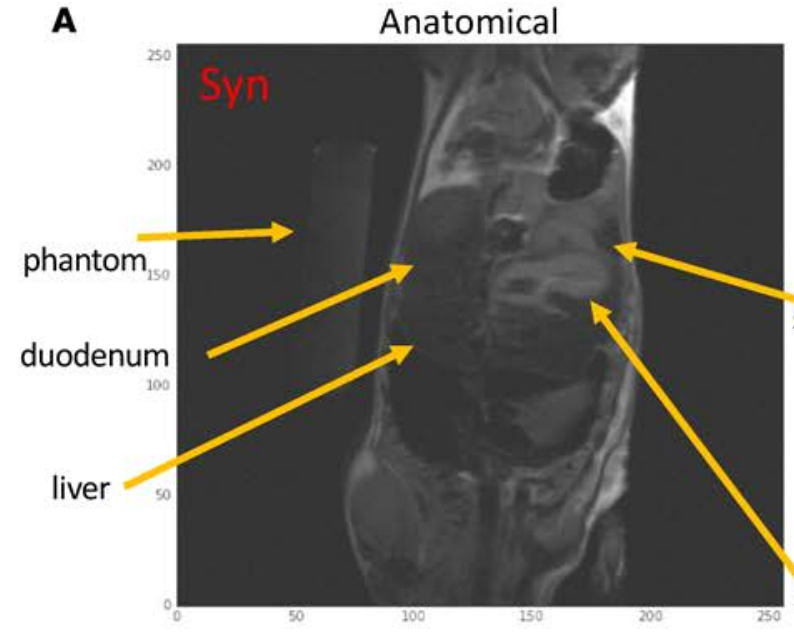

C

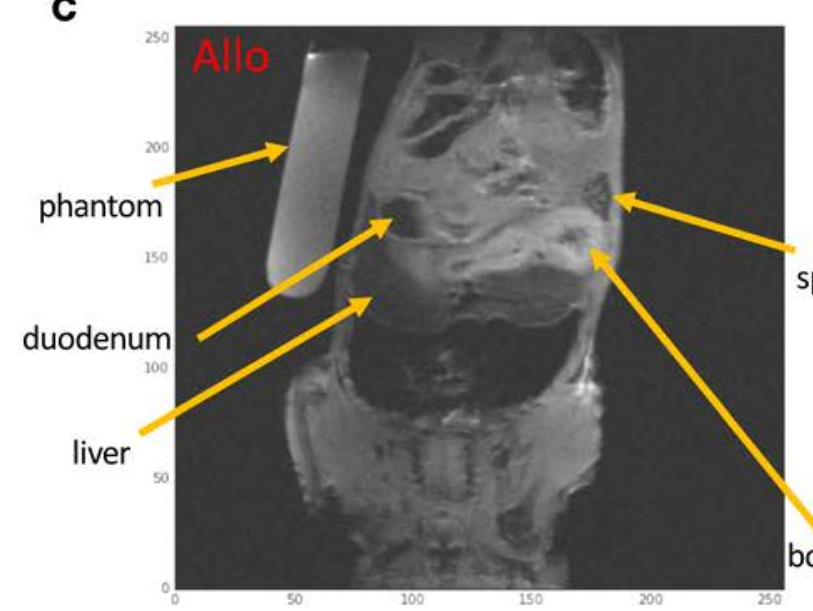

E

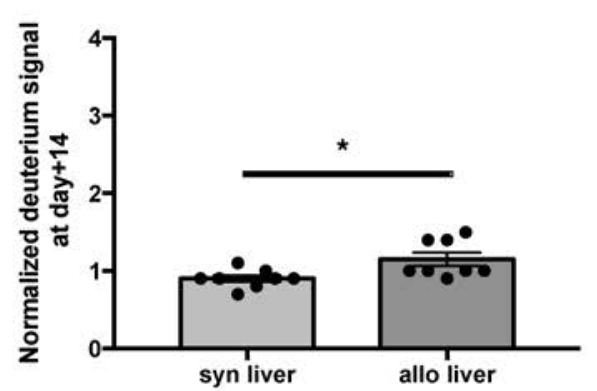

G

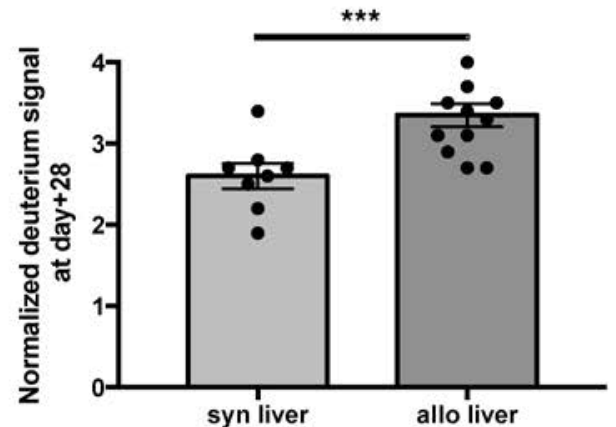

B

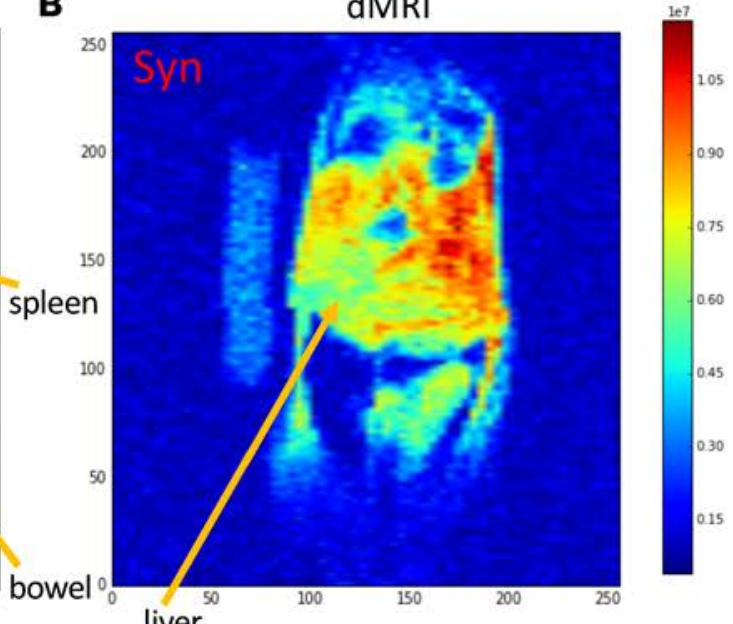

D

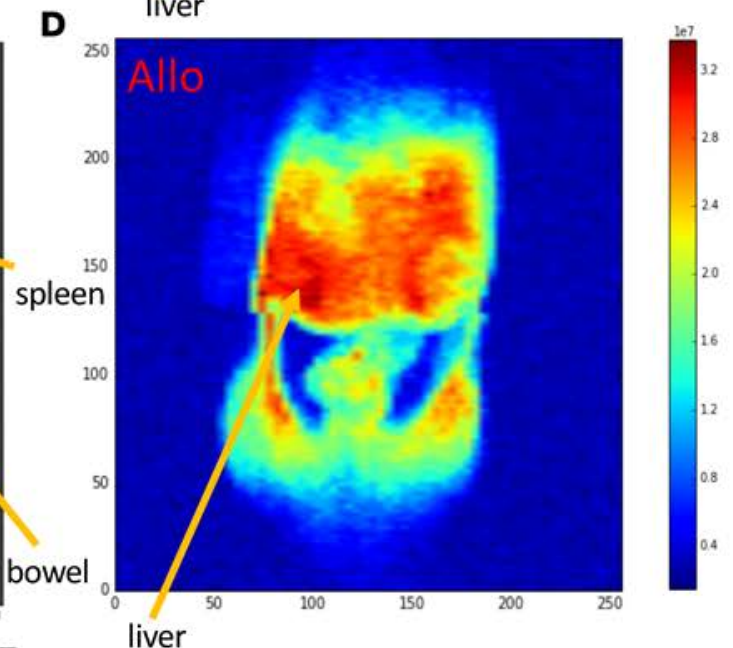

$\mathbf{F}$

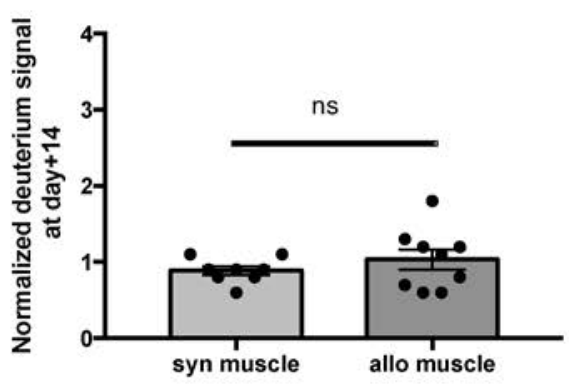

H

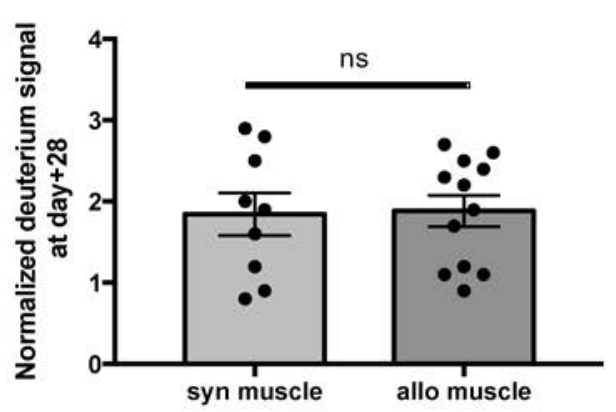

Figure 6. In vivo deuterium labeling followed by deuterium MRI (dMRI) facilitates diagnosis of chronic graft vs. host disease (cGVHD) in the target organ in allogeneic (allo) hematopoietic stem cell transplant (HSCT) recipients. (A and C) Proton (anatomical) MRI and dMRI (B and D) obtained with Bruker 9.7T magnet on day +28 following syngeneic HSCT (A and $\mathbf{B}$ ) and allogeneic HSCT (C and $\mathbf{D})$ after 21 days (day +7 to +28$)$ of ${ }^{2} \mathrm{H}_{2} \mathrm{O}$ labeling to total body water of $5 \%$. For each mouse, 3 coronal slices $3 \mathrm{~mm}$ in thickness were obtained through the midabdomen (covering liver and spleen); $5 \%{ }^{2} \mathrm{H}_{2} \mathrm{O}$ phantom was imaged to provide a reference deuterium signal. (E) Normalized deuterium signal (nDS) in the liver at day +14 was significantly 
higher in allogeneic recipients compared with syngeneic, while the unaffected tissue (muscle) nDS did not differ between cohorts, shown in $\mathbf{F}$. For $\mathbf{E}$ and $\mathbf{F}$, data are representative of 1 experiment ( $n=3$ per cohort). (G) nDS in the liver at day +28 was significantly higher in allogeneic recipients compared with syngeneic, while the muscle nDS did not differ between cohorts, shown in $\mathbf{H}$. For $\mathbf{G}$ and $\mathbf{H}$, data representative of 2 independent experiments ( $n=4$ and 3 for allo and syn, respectively). For panels E-H, ${ }^{*} P<0.05,{ }^{* *} P<0.001 ; 2$-sample, 2 -tailed, unequal variance $t$ test.

pathogenic $\mathrm{CD} 4^{+} \mathrm{T}$ cell subsets expand robustly following allogeneic HSCT. Our in vivo cell kinetics measurements concur with recent studies in auto- and alloimmunity that suggest Treg apoptosis may play a central role in regulating the size of the Treg niche $(58,59)$, especially in dysregulated immunity. However, we explored the dynamic behavior of Tregs in vivo through a methodological approach not previously applied to this subset. Our findings indicate that therapies for CGVHD should target Tem expansion, avoid harming Treg proliferation, and promote Treg survival. There have been several promising examples of the latter approach for acute GVHD and cGVHD, where Treg survival was improved either through manipulation of the cytokine milieu, for example low-dose IL-2 therapy $(12,13)$, or through affecting inherent changes in Tregs (60). To explore the mechanism/s for Treg apoptosis in cGVHD, our current work is focused on identifying metabolic differences between Treg, $\mathrm{T}_{\mathrm{N}}$, and Tem cells over the course of allogeneic HSCT. Cell kinetics studies described herein have formed the framework for this research, as they identified which cell subsets should be pursued, along with their anatomic location, their deuterium enrichment, and posttransplantation timing for further investigation.

By direct lymphocyte extraction $(61,62)$ from tissue parenchyma, we were able to map $\mathrm{CD} 4^{+} \mathrm{T}$ cell subset distribution to the relevant targets of cGVHD, including liver, bowel, and dermal skin, with the latter two not having been previously examined in the cGVHD setting with regard to $\mathrm{CD} 4^{+} \mathrm{T}$ cell subsets. Similar to patients with cGVHD, the allogeneic recipients in our model were lymphopenic when peripheral blood was evaluated. Likewise, low $\mathrm{CD} 4^{+} \mathrm{T}$ cell numbers were found in the thymus, lymph nodes, and spleen; however, these animals had a greatly increased number of $\mathrm{CD}^{+} \mathrm{T}$ cells in the target organs, particularly of the Tem phenotype. As shown in our in vivo deuterated water labeling experiments, the DNA of these rapidly proliferating $\mathrm{CD} 4^{+} \mathrm{Tem}$ cells becomes highly enriched with deuterium when deuterated water labeling is performed following HSCT. Combining the high deuterium enrichment and the contrasting distribution of Tem to cGVHD target organs in allogeneic versus syngeneic HSCT recipient animals, we hypothesized that cGVHD target organs could be visualized in vivo by MRI $(63,64)$. The feasibility of dMRI was described 3 decades ago, but dMRI has not been used in clinical or research settings due to lack of utility. However, we hypothesized that dMRI could be used to detect rapidly proliferating cells in vivo following brief in vivo deuterated water labeling, due to preferential incorporation of deuterium from body water by rapidly proliferating cells through the de novo nucleoside synthesis pathway. By manufacturing the deuterium-proton probe, we were able to entertain this hypothesis in the cGVHD preclinical model. We show that high enrichment of deuterium within target organs does indeed allow their visualization by dMRI. As hypothesized, dMRI provided a noninvasive means by which cGVHD animals could be distinguished from syngeneic counterparts. At day +14 , when allogeneic recipient mice are asymptomatic and have the same clinical scores as syngeneic controls (Supplemental Figure 1A), dMRI allowed noninvasive detection of cGVHD in target organs and also facilitated noninvasive in vivo diagnosis of cGVHD at day +28 with MRI images that were distinct between cGVHD-affected animals and syngeneic counterparts. In addition to DNA, other cellular components, i.e., lipids, proteins, peptides, amino acids, and metabolites, that are synthesized during the labeling period also incorporate deuterium, which may contribute to the high deuterium signal detected in target organs of cGVHD by dMRI. Nonetheless, dMRI allows nonradioactive and noninvasive imaging of cGVHD in the mouse model. Currently, no imaging modalities are diagnostic for cGVHD in patients. Hence, in vivo deuterium labeling followed by dMRI could serve as an early diagnostic tool for cGVHD, providing a body-wide assessment of disease involvement.

Given that deuterium enrichment is tied to cell proliferation, we demonstrate the first, to our knowledge, of many potential translational applications for this in vivo labeling-imaging approach. dMRI could allow noninvasive and nonradioactive imaging of many proliferating cell types, including neoplastic cells. As such, we have been able to image mouse tumor cells labeled with deuterium in vitro, and in vivo studies are planned. dMRI could provide an alternative or a complementary approach to current imaging modalities for cancer diagnosis and relapse surveillance, such as CT and/or PET, both of which involve radioactivity. In addition, it is possible that dMRI could be used for in vivo visualization of immunotherapeutic products shortly following infusion (e.g., chimeric antigen receptor $\mathrm{T}$ cells [CAR T cells], tumor 
infiltrating lymphocytes [TILs], and other adoptive immunotherapies), if such products undergo labeling during manufacture or expansion in culture prior to infusion into a patient.

In summary, the use of deuterated water labeling for dynamic measurement of in vivo cell kinetics and the imaging of such cells in vivo via dMRI should illuminate disease-specific pathophysiology and facilitate diagnosis of conditions characterized by rapidly dividing cells.

\section{Methods}

Mice

Three experimental cohorts were used: normal unmanipulated mice, recipients of syngeneic HSCT, and recipients of allogeneic HSCT. Female BALB/cAnNCr (H-2d) at 12-13 weeks of age served as recipients/hosts. For syngeneic transplantation, age-matched female BALB/c mice served as donors. In a subset of experiments, thymectomized BALB/c mice served as recipients, and for these, thymectomized animals were purchased. For allogeneic transplantation, age-matched female B10.D2-Hc1 H2-T18C/nSnJ $(\mathrm{H}-2 \mathrm{~d})$ mice served as donors. Congenic experiments were conducted using Thy1.1 B10.D2 and Thy1.1 Cg-FoxP3-GFP B10.D2 mice. $\mathrm{BALB} / \mathrm{c}$ mice were purchased from Charles River Laboratories. B10.D2 mice were purchased from The Jackson Laboratory. Congenic mice were bred at the NCI Frederick, MD breeding and holding facility.

\section{BM and splenocyte transplantation}

Recipient female mice were reconstituted with 15 million unfractionated splenocytes and 8 million BM cells injected via tail vein on day 0 , after 850 cGy total body irraditation (TBI) conditioning on day -1 , delivered in 2 divided doses 3 hours apart. Gentamicin was added to injection buffer (100 $\mu \mathrm{g} / \mathrm{ml})$. No additional antibiotics were administered following transplantation. Congenic experiments were conducted using an allogeneic graft composed of Thy1.1 Ly 9.2 spleen cells and Thy 1.2 Ly 9.2 T cell-depleted marrow transplanted into Thy1.2 Ly 9.1 hosts. Mice received regular drinking water and food until ${ }^{2} \mathrm{H}_{2} \mathrm{O}$ water labeling commenced. cGVHD scoring was performed twice weekly using previously described clinical scoring systems $(34,36)$. Animals were weighed weekly and on the day of euthanasia for experiments involving dermal and peripheral blood flow cytometry.

\section{${ }^{2} \mathrm{H}_{2} \mathrm{O}$ (deuterated water) labeling}

Deuterated water labeling was performed according to previously published protocol (38). Briefly, deuterated water was provided in drinking water after an initial i.v. bolus for specified labeling periods, during which newly synthesized cells incorporate deuterium into DNA bp. Incorporation of the label occurs through the de novo synthesis of nucleosides. Sequential labeling and pulse-chase experiments allow quantitative measurements of cell gain and cell loss, respectively, from an organ or tissue that the cells are extracted from. For cell gain kinetics, the fraction of newly synthesized cells during each 7-day labeling period was calculated by dividing $\mathrm{dA}$ $(\mathrm{M}+1)$ for the population of interest by $\mathrm{dA}(\mathrm{M}+1)$ for a reference fully turned-over population (unfractionated $\mathrm{BM})$. Unfractionated BM was collected for each experimental cohort as a pooled sample at each time point. Delabeling kinetics were obtained by measuring day $+14 \mathrm{dA}(\mathrm{M}+1)$ with a preceding 7-day labeling period. Thereafter, label administration ceased and mice received regular drinking water. Measurements for dA $(\mathrm{M}+1)$ were obtained at day $+21,+28$, and +35 for spleen delabeling experiments and day +21 and +28 for liver delabeling experiments. The enrichment values were then plotted over time. Formulas for delabeling curves were obtained in Microsoft Excel for Mac 2011 with linear, logarithmic, or polynomial fitting.

\section{Urine sample collection}

For each mouse that underwent $\mathrm{dMRI}, \sim 50 \mu \mathrm{l}$ of urine was collected prior to imaging. Urine collection was facilitated by placing the mouse on a strip of parafilm, then gently massaging the bilateral flanks. Upon spontaneous passage of urine onto parafilm, the urine was transferred into 1.5-ml Eppendorf with a transfer pipette. The urine samples were stored at $-20^{\circ} \mathrm{C}$ until TBW deuterium enrichment analysis on gas chromatography-tandem mass spectrometry (GC-MS/MS) was performed.

\section{Organ collection and preparation}

Integument. The harvesting of lymphocytes from flank sections was performed according to previously published methodology (61). Briefly, dorsal skin was harvested and s.c. adipose tissue was removed. Sections 
$\left(1 \mathrm{~cm}^{2}\right)$ underwent enzymatic digest in Liberase TL (Roche Diagnostics) for 2 hours at $37^{\circ} \mathrm{C}$ and $5 \% \mathrm{CO}_{2}$. Digested skin sections were then loaded into medicon cartridges and mechanically ground on the Medimachine System (Becton Dickinson). While lymphocytes were extracted from $1-\mathrm{cm}^{2}$ sections from each mouse, total body surface area (BSA) was calculated for each mouse based on weight (BSA = k mass 0.667, where $\mathrm{k}$ is the Meeh constant empirically determined for each species) (65), allowing whole skin lymphocyte content to be estimated.

Liver. Circulating nonparenchymal blood was flushed out of the liver prior to organ harvest by intracardiac injection of $20 \mathrm{ml}$ PBS with outflow through the cut portal vein. Gallbladder was removed prior to processing. Tissue was mechanically disrupted and sequentially filtered through $100 \mu \mathrm{m}, 70 \mu \mathrm{m}$, and finally $40 \mu \mathrm{m}$ filters (Thermo Fisher Scientific). Ammonium-chloride-potassium buffer lysis was used prior to resuspending cells for counting.

Small intestine. The small intestines were harvested and processed per previously described protocol (62). Briefly, the small intestine was cut proximally at the pyloric junction, then drawn out of the peritoneal cavity. Adipose tissue was manually removed. Another cut was made at the cecal junction, and the small intestine was then removed from the carcass. Intestinal tissue was placed into medium containing $3 \%$ FCS in RPMI (3\% media) on ice. Peyer's patches were then removed and processed separately (per lymphoid organ preparative procedure). The small intestine was cut longitudinally, and fecal matter was manually removed. Residual fecal material was rinsed off with 3\% media, followed by a rinse in HBSS. The tissue was then cut into $1-\mathrm{cm}$ sections and placed into solution containing $0.145 \mathrm{mg} / \mathrm{ml}$ DTT in $3 \%$ media and incubated for 20 minutes at $37^{\circ} \mathrm{C}$ with continuous mixing (magnetic stirrer mixing at $\sim 800 \mathrm{RPM}$ ). Following incubation, the contents were filtered through a stainless steel strainer. The suspension containing the intraepithelial lymphocytes (IEL) was placed on ice. The remaining tissue (on the strainer) was transferred into 0.5 M EDTA solution that was then vigorously shaken. Following this step, the solution was passed over the strainer, and the liquid portion was combined with the rest of IEL (subsequent preparation steps are described below).

Isolation of LP lymphocytes. The remaining intestinal sections were placed into solution containing $0.1 \mathrm{mg} / \mathrm{ml}$ Liberase TL (Roche Diagnostics) and $0.1 \mathrm{mg} / \mathrm{mL}$ DNase I (Sigma-Aldrich) and were finely minced. The mixture was then incubated for 30 minutes at $37^{\circ} \mathrm{C}$ with continuous stirring at $\sim 800 \mathrm{RPM}$. The solution was placed on ice, and $0.1 \mathrm{mg} / \mathrm{ml}$ DNase was added. The contents were then passed over a $70-\mu \mathrm{m}$ filter. The remnant intestinal pieces were crushed on the filter and rinsed with $0.1 \mathrm{mg} / \mathrm{ml} \mathrm{DNase}$ solution. The solution was then spun for 5 minutes at $4^{\circ} \mathrm{C}$ and $360 \mathrm{~g}$. The cell pellet was resuspended in $3 \%$ media and filtered over $40-\mu \mathrm{m}$ filter. Spin step was repeated and the cells were resuspended in $10 \%$ FCS in RPMI media for cell counting.

Isolation of IEL. The solution containing IEL was spun for 7 minutes at $4^{\circ} \mathrm{C}$ and $417 \mathrm{~g}$. The pellet was resuspended in $3 \%$ media and then filtered over $40-\mu \mathrm{m}$ filter, followed by another spin step. The pellet was then resuspended in 30\% Percoll solution (GE Healthcare) and spun for 20 minutes at room temperature and $545 \mathrm{~g}$. The cell pellet was resuspended in $0 \% \mathrm{FCS}$ media and spun for 7 minutes at $4^{\circ} \mathrm{C}$ and $417 \mathrm{~g}$. The remaining cell pellet was resuspended in 10\% FCS RPMI media for cell counting.

Lymphoid organs. Single-cell suspensions were obtained by mechanically disrupting lymphoid organs (thymus, spleen, and lymph nodes, including submandibular, axillary, inguinal, mesenteric, and Peyer's patches), then filtering through $70-\mu \mathrm{m}$ filters (Thermo Fisher Scientific). ACK lysis was performed on spleen samples to remove rbc prior to cell counting.

Peripheral blood. Blood was collected by orbital sinus cannulation with heparinized glass tubes and placed on ice. Total volume of the sample was recorded. Samples were then spun for 5 minutes at $4^{\circ} \mathrm{C}$ and 5,320 g. Serum was removed, and the samples underwent $2 \mathrm{ACK}$ lysis steps. Cells were then resuspended in media for counting. Total blood volume for each mouse based on $7 \%$ body weight was used to estimate total lymphocytes present in entire blood volume for each mouse by extrapolating lymphocyte numbers obtained from experimental samples of known collection volume.

\section{Staining for flow cytometry and fluorescence-activated cell sorting}

Cells were counted using Nexcelom Cellometer Auto T4 (Invitrogen) and Trypan Blue 0.4\% (Lonza). One million to 2 million cells were aliquoted for flow cytometry staining. For sorting, samples from multiple mice were pooled for each cohort (normal mice, syngeneic recipients, and allogeneic recipients). Surface antibody staining was performed on single-cell suspensions. The following antibodies were purchased from 
eBioscience, BD Biosciences, BioLegend, or Invitrogen: anti-active caspase-3 (BD Biosciences, 559341), anti-mouse CD4 (eBioscience, GK1.5), anti-mouse CD8 $\alpha$ (Invitrogen, 5H10, 53-6.7), anti-mouse CD8 $\beta$ (BD Biosciences), anti-mouse CD16/CD32 Fc block (BD Biosciences, 2.4G2), anti-mouse CD25 (eBioscience, PC61.5), anti-mouse CD69 (BioLegend, H1.2F3), anti-mouse/human CD44 (BioLegend, IM7), anti-CD90.2 (BD Biosciences, Thy1.2, 53-2.1), anti-mouse CD197/CCR7 (BioLegend, 4B12), antimouse/rat Foxp3 (eBioscience, FJK-16S), anti-mouse CD229.1 (BD Bioscience, Ly9.1, 30C7), Streptavidin Pacific Blue (Invitrogen, S-11222), anti-mouse $\gamma \delta$ TCR (eBioscience, eBioGL3), anti-TCR $\beta$ (BD Biosciences, H57-597). LIVE/DEAD Fixable Aqua Dead Cell Stain Kit (Invitrogen) was used to exclude dead cells. mCD1d (PBS-57) Tetramer was obtained from NIH Tetramer Core Facility for staining liver parenchyma, to allow exclusion of NKT cells from T cells for FACS and flow cytometry phenotyping. The cells were fixed with eBioscience fixation/permeabilization reagents, and then intracellular staining for Foxp3 was performed overnight at $4^{\circ} \mathrm{C}$. Pooled samples for each cohort underwent FACS using BD Influx, with $95 \%$ purity. Individual mouse sample flow cytometry measurements were obtained on BD LSR II, and collected data were analyzed using FlowJo 9.7.6 Software. FACS-purified samples were collected into PBS buffer containing $2 \%$ BSA. The samples were spun for 10 minutes at $4^{\circ} \mathrm{C}$ and $10,060 \mathrm{~g}$. Supernatant was removed, and remaining cell pellets were stored at $-80^{\circ} \mathrm{C}$ until DNA extraction.

DNA extraction. DNA extraction from nonfixed cells was performed on the Promega Maxwell 16 system as previously described (38). For DNA extraction from sorted fixed cells, EpiSonic 1100 Sonication System was used.

\section{GC-MS/MS analysis}

$d A$ enrichment measurements. Quantitative determination of $\mathrm{dA}$, its isotopologue $(\mathrm{dA}[\mathrm{M}+1])$ and the internal standard (dA M+5) was measured using validated GC-MS/MS methodology (38). Briefly, DNA extracted from FACS purified T cell subsets was hydrolyzed to its bp using EpiQuick DNA Hydrolysis Kit (EpiGentek). The bp were then purified and concentrated using solid phase extraction (SPE). The SPE extracts were dried under vacuum, and MethElute (methylation reagent, Thermo Fisher Scientific) was added to the residue and mixed thoroughly. The Agilent 7890A GC, LTM series II fast GC module, 7000A triple quadrupole GC-MS, and 7693 autosampler were then used. Upon injection into the GC, the derivatized bp were separated using low thermal mass fast GC. Calibration standards of $\mathrm{dA}, \mathrm{dA}(\mathrm{M}+1)$, and the internal standard ( $\mathrm{AA} M+5)$ were used for quantitative MS, utilizing positive chemical ionization and the MRM mode of MS detection.

$T B W$ enrichment measurements. For measuring ${ }^{2} \mathrm{H}_{2} \mathrm{O}$ water levels in TBW (e.g., urine), we developed a simple and quantitative headspace GC-NCI-MS method (66). Briefly, the method utilizes a rapid gas phase isotopic exchange of the ${ }^{1} \mathrm{H}:{ }^{2} \mathrm{H}$ moiety between ${ }^{2} \mathrm{H}_{2} \mathrm{O}$ water in $\mathrm{TBW}$ and the acetone solvent used for isotopic exchange. The method requires $25 \mu 1$ of TBW sample, i.e. urine; demonstrates a linear relationship from $2 \%-40 \%(\mathrm{v} / \mathrm{v}){ }^{2} \mathrm{H}_{2} \mathrm{O}$ in TBW; and has a total analysis time of less than 10 minutes.

\section{Histopathology}

Thymus, lymph nodes, spleen, liver, stomach, small intestine, colon, skin, lung, and BM (sternum) were extracted from mice immediately following euthanasia and placed into $4 \% \mathrm{w} / \mathrm{v}$ formaldehyde. Tissue cassettes were then sent to Histoserv Inc. for sectioning and $\mathrm{H} \& \mathrm{E}$ staining. $\mathrm{H} \& \mathrm{E}$ slides were evaluated for $\mathrm{CGVHD}$ histological grading by M.A. Eckhaus. Adobe Photoshop Elements 8.0 was used to acquire images of the $\mathrm{H} \&$ E figures obtained via Olympus DP12 camera, visualized through an Olympus BX41 microscope (magnification as noted on each figure).

\section{Proton and dMRI}

All MRI experiments were performed on a 9.4 Tesla magnet equipped with a Bruker Advance III MRI console (Bruker-Biospin). Immediately following euthanasia, each mouse was wrapped in plastic and taped onto a plastic cradle in a flat, level position. A 5-mm diameter tube (phantom) containing $5 \%{ }^{2} \mathrm{H}_{2} \mathrm{O}$ in ddH $\mathrm{O}_{2} \mathrm{O}$ with $0.1 \%$ sodium azide (preservative) was placed adjacent to the mouse as a reference and calibration standard. The cradle was centered in the MRI probe described above and then placed in the magnet. Following acquisition of a set of standard locator images, a set of 3 coronal planes were prescribed covering the spleen and liver. Reference images of these regions were acquired using the multislice multiecho sequence and the following parameters: field of view, $40 \times 40 \mathrm{~mm}$; slice thickness, $1 \mathrm{~mm}$; repetition time/echo time (TR/TE), 1,000/14 
$\mathrm{ms}$; and the matrix of $256 \times 256$. Subsequently, the same planes were imaged using the deuterium sequence with the following parameters: field of view, $40 \times 40 \mathrm{~mm}$; slice thickness, $3 \mathrm{~mm}$; TR/TE, 398/1.6 ms; spatial matrix, $128 \times 64$; and spectral matrix, 512. The excitation flip angle was adjusted to meet an Ernst angle condition. All imaging data were analyzed using custom code written in Python. Following spectroscopic reconstruction, regions of interest were placed onto the reference sample, the spleen (when visible), the liver, and muscle. The average intensity and the SD of the intensity was measured and tabulated for each region on the deuterium and reference images. Anatomical regions were normalized using the reference $\left(5 \%{ }^{2} \mathrm{H}_{2} \mathrm{O}\right.$ phantom) intensity to compensate for experimental variation.

\section{${ }^{1} \mathrm{H}-{ }^{2} \mathrm{H}$, proton-deuterium, coil for MRI}

The schematic for the components of the proton-deuterium coil is provided in Supplemental Figure 7.

\section{Statistics}

Data for experimental cohorts were graphed with Prism 6.0 (GraphPad Software Inc.) or Microsoft Excel for Mac 2011. Error bars on bar graphs represented \pm SEM. For monovariate data Microsoft Excel for Mac 2011 was used, and 2-sample, 2-tailed, unequal variance $t$ tests were performed. When applicable, a 2-way ANOVA analysis of variance was performed to determine interaction effect, followed by post hoc Tukey Kramer HSD pairwise analysis. Minitab 16.2.4 was used to perform a portion of analyses. ${ }^{*} P<$ $0.05,{ }^{* *} P<0.01$, and ${ }^{* * *} P<0.001$

\section{Study Approval}

The present studies conducted on animals in the Clinical Research Center (CRC) and Mouse Imaging Facility (MIF) under the EIB-020 protocol were reviewed and approved by the NCI, NIH, Animal Care and Use Committee (ACUC).

\section{Author Contributions}

NPB designed and conducted the experiments, analyzed and interpreted data, and wrote the manuscript. DEF contributed to dMRI experiments, conducted GC-MS/MS experiments, and analyzed GC-MS/MS data. NM assisted with experiments, data analysis, and manuscript revisions. ML performed MRI experiments. HM designed and made the deuterium-proton coil. BUO assisted NPB with experiments. ACC and RJB contributed to small intestinal lymphocyte experiments. VK performed FACS purifications. EC performed injections (i.p. and i.v.) on mice. GAS assisted with a subset of nonimaging experiments and statistical analysis. LMDS, NJB, and YB contributed to skin lymphocyte experiments. FAF made significant contributions to manuscript revision. WGT contributed to FACS experimental design. MAE performed histological cGVHD scoring of mouse organs. CVB assisted in the procurement of EpiSonic 1100 Sonication System and the Agilent GC-headspace unit, as well as maintained ACUC approval of animal studies. REG contributed to design of the experiments and sponsored the research.

\section{Acknowledgments}

We thank the NIH Tetramer Facility for providing the CD1d PBS-57 tetramer, which allowed us to extract pure $\mathrm{CD}^{+} \mathrm{T}$ cell populations from liver samples. We acknowledge Ethan Tyler (Medical Illustrator, NIH) for making a schematic figure of $\mathrm{T}$ cell distribution for the manuscript. We thank Kevin $\mathrm{Hu}$ (NIH Summer Internship Program and Eastern Virginia Medical School) for assisting with organization of the manuscript layout. We are grateful to Carole Sourbier, NCI, for editing and insightful manuscript suggestions. We acknowledge Brenda Klaunberg of Mouse Imaging Facility (MIF), NIH, for facilitating the imaging that was conducted within the MIF. Our work was supported by NCI, NIH, intramural funding.

Address correspondence to: Nataliya P. Buxbaum, Experimental Transplantation and Immunology Branch, National Cancer Institute, National Institutes of Health, Building 10 CRC, Room 3-3288, 10 Center Drive, Bethesda, Maryland 20892-1104,USA. Phone: 301.435.3547; Email: nbuxbaum@mail.nih.gov.

\footnotetext{
1. Sakaguchi S, Sakaguchi N, Asano M, Itoh M, Toda M. Immunologic self-tolerance maintained by activated T cells expressing IL-2 receptor alpha-chains (CD25). Breakdown of a single mechanism of self-tolerance causes various autoimmune dis eases. J Immunol. 1995;155(3):1151-1164.
} 
2. Bluestone JA, Herold K, Eisenbarth G. Genetics, pathogenesis and clinical interventions in type 1 diabetes. Nature. 2010;464(7293):1293-1300.

3. Sakaguchi S, Yamaguchi T, Nomura T, Ono M. Regulatory T cells and immune tolerance. Cell. 2008;133(5):775-787.

4. Belkaid Y, Rouse BT. Natural regulatory T cells in infectious disease. Nat Immunol. 2005;6(4):353-360.

5. Lund JM, Hsing L, Pham TT, Rudensky AY. Coordination of early protective immunity to viral infection by regulatory T cells. Science. 2008;320(5880):1220-1224.

6. Aandahl EM, Michaëlsson J, Moretto WJ, Hecht FM, Nixon DF. Human CD4+ CD25+ regulatory T cells control T-cell responses to human immunodeficiency virus and cytomegalovirus antigens. J Virol. 2004;78(5):2454-2459.

7. Gajewski TF, Schreiber H, Fu YX. Innate and adaptive immune cells in the tumor microenvironment. Nat Immunol. 2013;14(10):1014-1022.

8. Gattinoni L, Klebanoff CA, Restifo NP. Paths to stemness: building the ultimate antitumour T cell. Nat Rev Cancer. 2012;12(10):671-684.

9. Shlomchik WD. Graft-versus-host disease. Nat Rev Immunol. 2007;7(5):340-352.

10. Ferrara JL, Levine JE, Reddy P, Holler E. Graft-versus-host disease. Lancet. 2009;373(9674):1550-1561.

11. Lee SJ, Vogelsang G, Flowers ME. Chronic graft-versus-host disease. Biol Blood Marrow Transplant. 2003;9(4):215-233.

12. Matsuoka K, et al. Low-dose interleukin-2 therapy restores regulatory T cell homeostasis in patients with chronic graft-versushost disease. Sci Transl Med. 2013;5(179):179ra43

13. Koreth J, et al. Interleukin-2 and regulatory T cells in graft-versus-host disease. N Engl J Med. 2011;365(22):2055-2066

14. McDonald-Hyman C, et al. Therapeutic regulatory T-cell adoptive transfer ameliorates established murine chronic GVHD in a CXCR5-dependent manner. Blood. 2016;128(7):1013-1017.

15. Di Ianni M, et al. Tregs prevent GVHD and promote immune reconstitution in HLA-haploidentical transplantation. Blood. 2011;117(14):3921-3928.

16. Busch R, Neese RA, Awada M, Hayes GM, Hellerstein MK. Measurement of cell proliferation by heavy water labeling. Nat Protoc. 2007;2(12):3045-3057.

17. Cohen A, Barankiewicz J, Lederman HM, Gelfand EW. Purine and pyrimidine metabolism in human T lymphocytes. Regulation of deoxyribonucleotide metabolism. J Biol Chem. 1983;258(20):12334-12340.

18. Hellerstein M, et al. Directly measured kinetics of circulating T lymphocytes in normal and HIV-1-infected humans. Nat Med. 1999;5(1):83-89.

19. Hellerstein MK, et al. Subpopulations of long-lived and short-lived T cells in advanced HIV-1 infection. J Clin Invest. 2003;112(6):956-966.

20. Calissano C, et al. In vivo intraclonal and interclonal kinetic heterogeneity in B-cell chronic lymphocytic leukemia. Blood. 2009; $114(23): 4832-4842$.

21. Calissano C, et al. Intraclonal complexity in chronic lymphocytic leukemia: fractions enriched in recently born/divided and older/quiescent cells. Mol Med. 2011;17(11-12):1374-1382.

22. Hayes GM, et al. Isolation of malignant B cells from patients with chronic lymphocytic leukemia (CLL) for analysis of cell proliferation: validation of a simplified method suitable for multi-center clinical studies. Leuk Res. 2010;34(6):809-815.

23. Hayes GM, et al. Regional cell proliferation in microdissected human prostate specimens after heavy water labeling in vivo: correlation with prostate epithelial cells isolated from seminal fluid. Clin Cancer Res. 2012;18(12):3250-3260.

24. Messmer BT, et al. In vivo measurements document the dynamic cellular kinetics of chronic lymphocytic leukemia B cells. J Clin Invest. 2005;115(3):755-764.

25. Chen S, et al. Measurement of pancreatic islet cell proliferation by heavy water labeling. Am J Physiol Endocrinol Metab. 2007;293(5):E1459-E1464.

26. Tchoukalova YD, et al. In vivo adipogenesis in rats measured by cell kinetics in adipocytes and plastic-adherent stroma-vascular cells in response to high-fat diet and thiazolidinedione. Diabetes. 2012;61(1):137-144

27. Bollyky JB, et al. Evaluation of in vivo T cell kinetics: use of heavy isotope labelling in type 1 diabetes. Clin Exp Immunol. 2013;172(3):363-374.

28. Shankaran M, et al. Measurement of brain microglial proliferation rates in vivo in response to neuroinflammatory stimuli: application to drug discovery. J Neurosci Res. 2007;85(11):2374-2384.

29. Li KW, Siraj SA, Cheng EW, Awada M, Hellerstein MK, Turner SM. A stable isotope method for the simultaneous measurement of matrix synthesis and cell proliferation in articular cartilage in vivo. Osteoarthr Cartil. 2009;17(7):923-932.

30. Lahoz-Beneytez J, et al. Human neutrophil kinetics: modeling of stable isotope labeling data supports short blood neutrophil half-lives. Blood. 2016;127(26):3431-3438.

31. Jagasia MH, et al. National Institutes of Health Consensus Development Project on Criteria for Clinical Trials in Chronic Graft-versus-Host Disease: I. The 2014 Diagnosis and Staging Working Group report. Biol Blood Marrow Transplant. 2015;21(3):389-401.e1.

32. Filipovich AH, et al. National Institutes of Health consensus development project on criteria for clinical trials in chronic graftversus-host disease: I. Diagnosis and staging working group report. Biol Blood Marrow Transplant. 2005;11(12):945-956.

33. Chu YW, Gress RE. Murine models of chronic graft-versus-host disease: insights and unresolved issues. Biol Blood Marrow Trans plant. 2008;14(4):365-378.

34. Anderson BE, McNiff JM, Matte C, Athanasiadis I, Shlomchik WD, Shlomchik MJ. Recipient CD4+ T cells that survive irradiation regulate chronic graft-versus-host disease. Blood. 2004;104(5):1565-1573.

35. Reddy P, Ferrara JLM. Mouse models of graft-versus-host disease. StemBook. Cambridge (MA): Harvard Stem Cell Institute; 2008.

36. Anderson BE, et al. Memory CD4+ T cells do not induce graft-versus-host disease. J Clin Invest. 2003;112(1):101-108.

37. Reddy P, Negrin R, Hill GR. Mouse models of bone marrow transplantation. Biol Blood Marrow Transplant. 2008;14(1 Suppl 1):129-135

38. Farthing DE, et al. Sensitive GC-MS/MS method to measure deuterium labeled deoxyadenosine in DNA from limited mouse cell populations. Anal Chem. 2013;85(9):4613-4620. 
39. Marmur J, Doty P. Determination of the base composition of deoxyribonucleic acid from its thermal denaturation temperature. J Mol Biol. 1962;5:109-118.

40. Meinke W, Goldstein DA, Hall MR. Rapid isolation of mouse DNA from cells in tissue culture. Anal Biochem. 1974;58(1):82-88.

41. Matthews $\mathrm{K}$, et al. Imbalance of effector and regulatory CD4 T cells is associated with graft-versus-host disease after hematopoietic stem cell transplantation using a reduced intensity conditioning regimen and alemtuzumab. Haematologica. 2009;94(7):956-966.

42. Alho AC, et al. Unbalanced recovery of regulatory and effector T cells after allogeneic stem cell transplantation contributes to chronic GVHD. Blood. 2016;127(5):646-657.

43. Nguyen VH, et al. In vivo dynamics of regulatory T-cell trafficking and survival predict effective strategies to control graft-versus-host disease following allogeneic transplantation. Blood. 2007;109(6):2649-2656.

44. Chen X, Vodanovic-Jankovic S, Johnson B, Keller M, Komorowski R, Drobyski WR. Absence of regulatory T-cell control of TH1 and TH17 cells is responsible for the autoimmune-mediated pathology in chronic graft-versus-host disease. Blood. 2007;110(10):3804-3813.

45. Koreth J, et al. Efficacy, durability, and response predictors of low-dose interleukin-2 therapy for chronic graft-versus-host disease. Blood. 2016;128(1):130-137.

46. Dulude G, Roy DC, Perreault C. The effect of graft-versus-host disease on T cell production and homeostasis. J Exp Med. 1999;189(8):1329-1342.

47. Nguyen $\mathrm{VH}$, et al. The impact of regulatory T cells on T-cell immunity following hematopoietic cell transplantation. Blood. 2008;111(2):945-953.

48. Beilhack A, et al. In vivo analyses of early events in acute graft-versus-host disease reveal sequential infiltration of T-cell subsets. Blood. 2005;106(3):1113-1122

49. Beilhack A, et al. Prevention of acute graft-versus-host disease by blocking T-cell entry to secondary lymphoid organs. Blood 2008;111(5):2919-2928.

50. Busch R, Siah IM, Gee TA, Hellerstein MK. Heavy water labeling of DNA for measurement of cell proliferation and recruitment during primary murine lymph node responses against model antigens. J Immunol Methods. 2008;337(1):24-34.

51. Ladell K, Hellerstein MK, Cesar D, Busch R, Boban D, McCune JM. Central memory CD8+ T cells appear to have a shorter lifespan and reduced abundance as a function of HIV disease progression. J Immunol. 2008;180(12):7907-7918.

52. Li KW, Turner SM, Emson CL, Hellerstein MK, Dale DC. Deuterium and neutrophil kinetics. Blood. 2011;117(22):6052-3; author reply 6053.

53. McCune JM, et al. Factors influencing T-cell turnover in HIV-1-seropositive patients. J Clin Invest. 2000;105(5):R1-R8

54. Neese RA, et al. Measurement in vivo of proliferation rates of slow turnover cells by $2 \mathrm{H} 2 \mathrm{O}$ labeling of the deoxyribose moiety of DNA. Proc Natl Acad Sci USA. 2002;99(24):15345-15350.

55. Imanguli MM, et al. Comparative analysis of FoxP3(+) regulatory $\mathrm{T}$ cells in the target tissues and blood in chronic graft versus host disease. Leukemia. 2014;28(10):2016-2027.

56. Sakoda Y, et al. Donor-derived thymic-dependent T cells cause chronic graft-versus-host disease. Blood. 2007;109(4):1756-1764.

57. Zhang Y, Hexner E, Frank D, Emerson SG. CD4+ T cells generated de novo from donor hemopoietic stem cells mediate the evolution from acute to chronic graft-versus-host disease. J Immunol. 2007;179(5):3305-3314.

58. Pierson W, et al. Antiapoptotic Mcl-1 is critical for the survival and niche-filling capacity of Foxp $3^{+}$regulatory T cells. Nat Immunol. 2013;14(9):959-965.

59. Shen CR, Yang WC, Chen HW. The fate of regulatory T cells: survival or apoptosis. Cell Mol Immunol. 2014;11(1):11-13.

60. Aoyama K, et al. Inhibiting retinoic acid signaling ameliorates graft-versus-host disease by modifying T-cell differentiation and intestinal migration. Blood. 2013;122(12):2125-2134.

61. Naik S, et al. Compartmentalized control of skin immunity by resident commensals. Science. 2012;337(6098):1115-1119.

62. Sun CM, et al. Small intestine lamina propria dendritic cells promote de novo generation of Foxp3 T reg cells via retinoic acid. JExp Med. 2007;204(8):1775-1785.

63. Müller S, Seelig J. In Vivo NMR Imaging of Deuterium. J Magn Reson. 1969;72(3): 456-466.

64. Ewy C, Babcock EE, Ackerman JJH. Deuterium Nuclear Magnetic Resonance Spin-Imaging of D2O: A Potential Exogenous MRI Label. Magn Reson Imaging. 1986;4(5): 407-411.

65. Cheung MC, et al. Body surface area prediction in normal, hypermuscular, and obese mice. J Surg Res. 2009;153(2):326-331.

66. Farthing DE, et al. Comparing DNA enrichment of proliferating cells following administration of different stable isotopes of heavy water. Sci Rep. In press. 